\title{
The Framed Morse Complex and its Invariants
}

\author{
S. A. BARANNIKOV
}

\section{Introduction}

Consider a smooth function $f$ defined in a neighborhood of the boundary of an $n$-dimensional ball. A smooth continuation of the function to the interior of the ball has generically only nondegenerate critical points. We shall obtain a lower bound for the number of critical points similar to the one Betti numbers give for the number of critical points of a function on a closed manifold.

Our approach is based on studying certain geometric invariants of the given function. They can be constructed from the following information:

(1) the Morse complex of the restriction of the given function to the sphere bounding the ball;

(2) the critical values of $\left.f\right|_{S^{n-1}}$;

(3) the directions of the gradient of the function at the critical points of $\left.f\right|_{S^{n-1}}$ (inside or outside the ball).

When the sphere moves to the interior of the ball, this information changes in a specific way (see $\S 1$ ). So do our invariants. From these facts we get a lower bound for the number of the critical points.

The word "invariant" means that this structure does not depend on a metric of the sphere. Similar invariants can be constructed for functions on arbitrary manifolds.

In $\S 1$ we study the behavior of the data described above. In $\S 2$ we introduce our invariants and explain how they yield a lower bound for the number of the critical points. In $\S 3$ we describe an application of similar invariants to functions on arbitrary manifolds.

I am grateful to V. I. Arnold for formulation of the problem and helpful discussions, and to V. A. Vassiliev for many important remarks and interest in this work. 


\section{$\S 1$. The framed Morse complex}

1.1. Definition of the framed Morse complex. The usual Morse complex is defined for a generic smooth function and a metric on a closed manifold in the following way [2, $4,7,8]$. Its $k$-dimensional chain group is generated over $\mathbb{Z}$ by index $k$ critical points of the function. The boundary map $d: C_{k} \rightarrow C_{k-1}$ is defined by the formula $d p=\sum \alpha_{i} q_{i}$ where $\alpha_{i}$ is the algebraic number of gradient curves connecting a critical point $p$ of index $k$ with the critical point $q_{i}$ of index $(k-1)$.

We fix a Riemannian metric on the $n$-dimensional ball (and hence on any sphere embedded in the ball).

Definition. A framed Morse complex is the following information on a generic smooth function $f$ on a neighborhood of the boundary of a compact Riemannian manifold $M$ :

(1) the Morse complex of the restriction of the given function to the boundary $\partial M$ of the manifold;

(2) the critical values of $\left.f\right|_{\partial M}$;

(3) the directions of the gradient of the function at critical points of $\left.f\right|_{\partial M}$ (inside or outside the manifold $M$ ).

\subsection{Definition of the abstract framed Morse complex.}

Definition. An $n$-dimensional abstract framed Morse complex is the following structure:

(1) A finite set of points on $n$ real lines numbered by indices $0,1, \ldots$, $(n-1)$. (The lines are represented on our figures by vertical lines). We call these points heights. The highest height is on the line of index $(n-1)$. The lowest height is on the line of index 0 .

(2) The chain complex $\left(C_{*}, d\right)$ of $\mathbb{Z}$-modules $C_{k}$ generated by the heights of index $k$. The differential $d$ respects the following condition: if $d a=$ $\sum \alpha_{i} b_{i}, \alpha_{i} \in \mathbb{Z}, \alpha_{i} \neq 0$, then the height $a$ is higher then any height $b_{i}$.

(3) Each height has direction up or down (which is indicated by an arrow on our figures).

The abstract framed Morse complex can be represented as in Figure 1.

We put the heights with their directions on the corresponding lines. We connect the height $a$ with the heights $b_{i}$ by the line segment if $d a=\sum \alpha_{i} b_{i}$, $\alpha_{i} \in \mathbb{Z}, \alpha_{i} \neq 0$, and we write the number $\alpha_{i}$ on the segment connecting $a$ with $b_{i}$. If this number is 1 , we write nothing.

We abbreviate "abstract framed Morse complex" to "abstract FMC." We denote by $\tilde{a}$ the height corresponding to a generator $a$ of the complex.

Associate an abstract FMC with any framed Morse complex of a generic smooth function on the neighborhood of the boundary of an $n$-dimensional ball in the following way. The heights correspond to the critical values of the critical points of the function. The directions of the heights correspond to the directions in which the critical values move if the sphere moves inside the ball. 

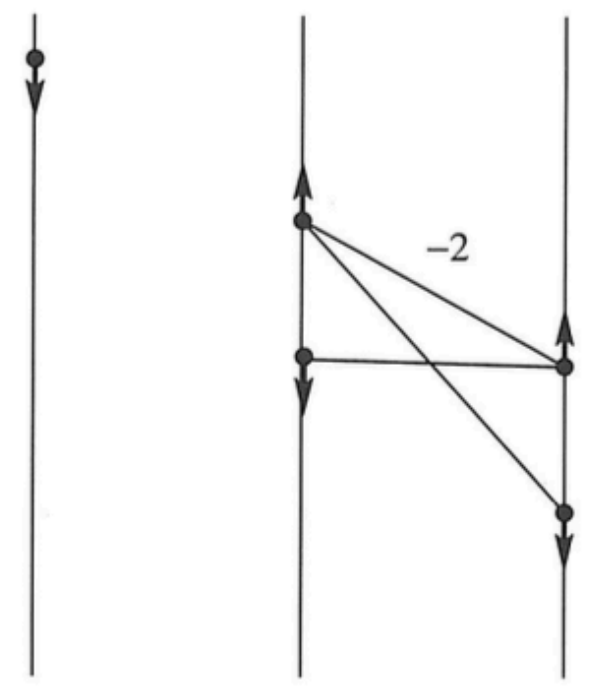

$\mid$

FIGURE 1. An abstract framed Morse complex

1.3. Metamorphoses of the abstract framed Morse complex. We shall consider the following metamorphoses of the abstract FMC:

(1) Change of complex (Figure 2). Let $\tilde{a}$ and $\tilde{b}$ be two heights having the same index $k, \tilde{a}<\tilde{b}$. Consider the chain groups generated by heights. The change of complex is described as the change of generators $b \rightarrow b \pm a$ in the $k$-dimensional chain group. We assume that the differentials in the chain groups are invariant with respect to the choice of basis.

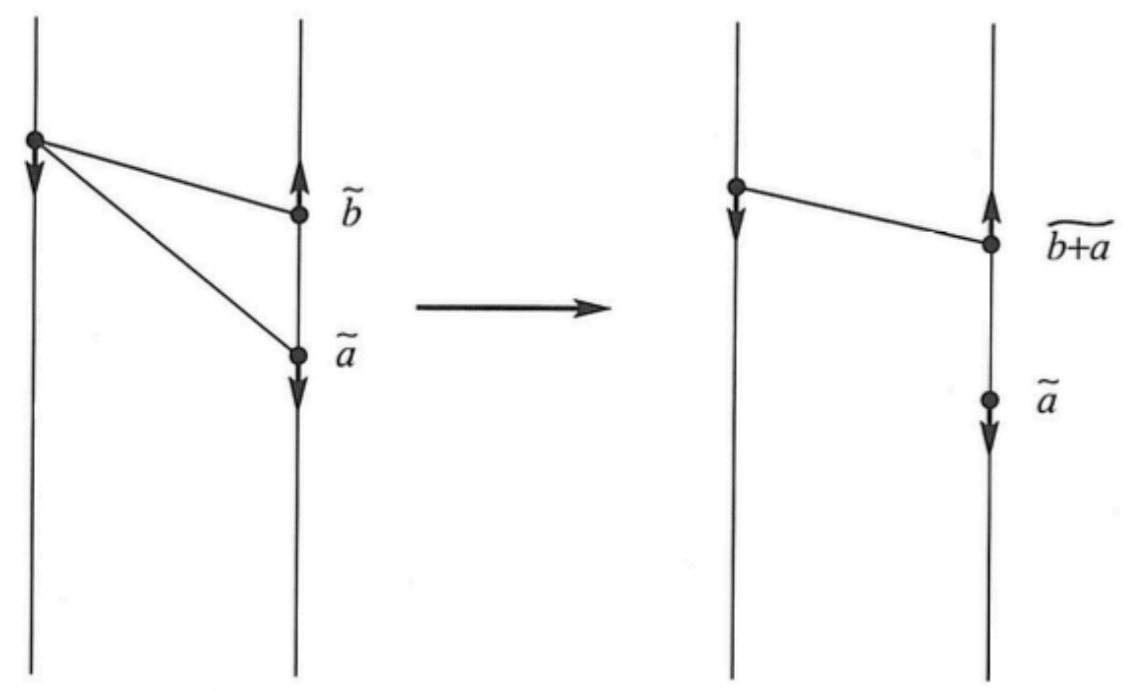

FIGURE 2

(2a) Birth of a pair of heights. Birth of a pair $\tilde{a}$ and $\tilde{b}$ of neighboring heights such that $d a=b$ and the heights have the same directions (Figure 3).

(2b) Death of a pair of heights. Death of the same pair of heights as in metamorphosis 2a (Figure 3).

(3a) Shift of heights. Shift of the heights along their directions without meeting a pair of heights of the same index (Figure 4). 


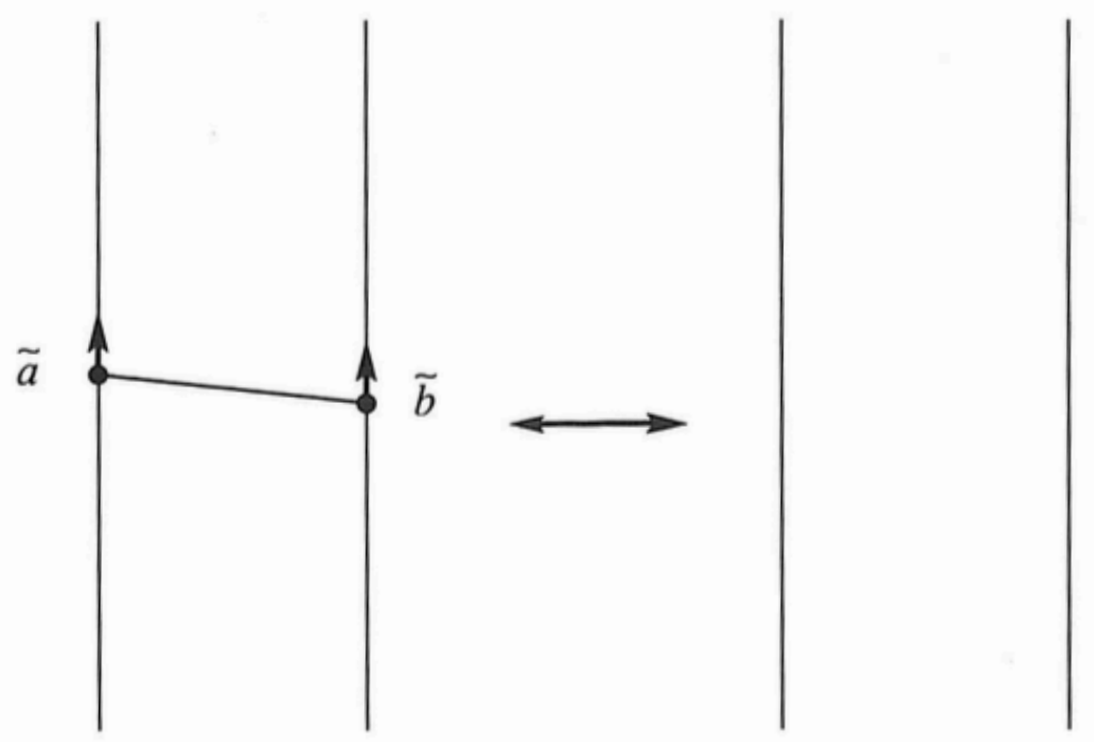

FIGURE 3
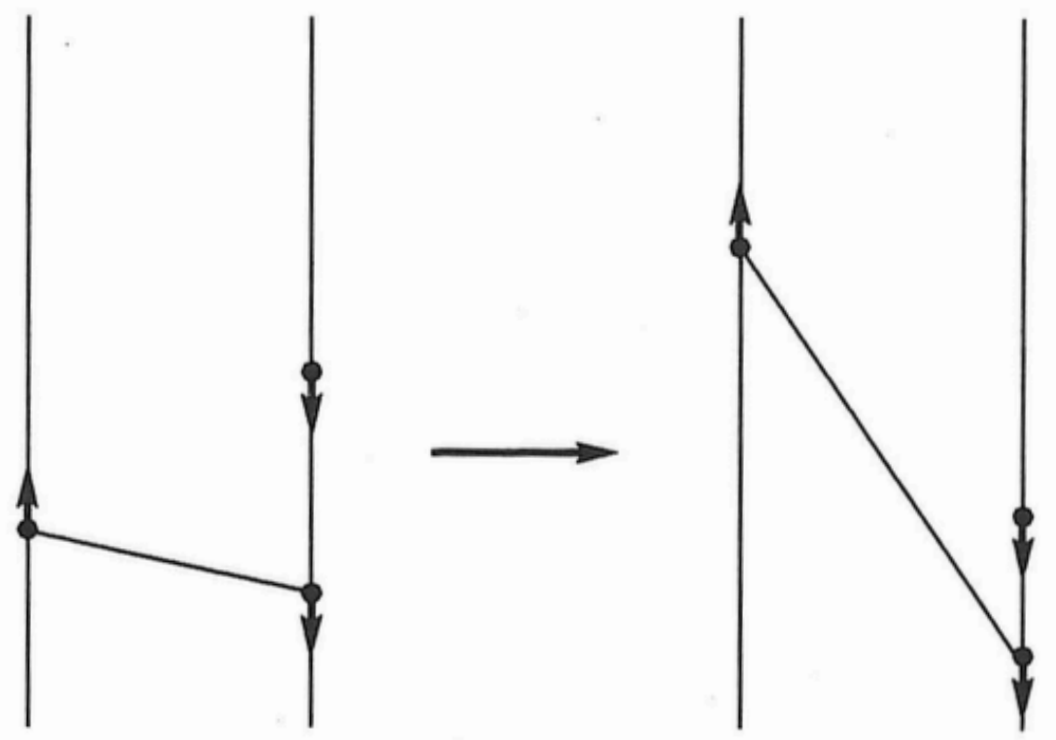

FIGURE 4

(3b) Passing a height by another height. Passing a height by another height of the same index as they move along their directions (Figure 5).

Metamorphoses $3 \mathrm{a}$ and $3 \mathrm{~b}$ respect the following condition: if there is a nonzero term in $d a=\sum \alpha_{i} b_{i}$ corresponding to a height $\tilde{b}$, then the height $\tilde{a}$ stays higher than $\tilde{b}$.

(4) Switching the direction of a height. These metamorphoses have indices. A metamorphosis of index $\lambda$ is the switch in the direction of a height of index $\lambda-1$ from up to down or the direction of a height of index $\lambda$ from down to up. (Figure 6.)

Suppose that a function defined in a neighborhood of the boundary of a ball has been extended to a continuous function in the interior of the ball with several nondegenerate critical points. Move the sphere to which we restrict the given function to the interior of the ball.

THEOREM 1. If, in the process of its motion, the sphere does not intersect the 

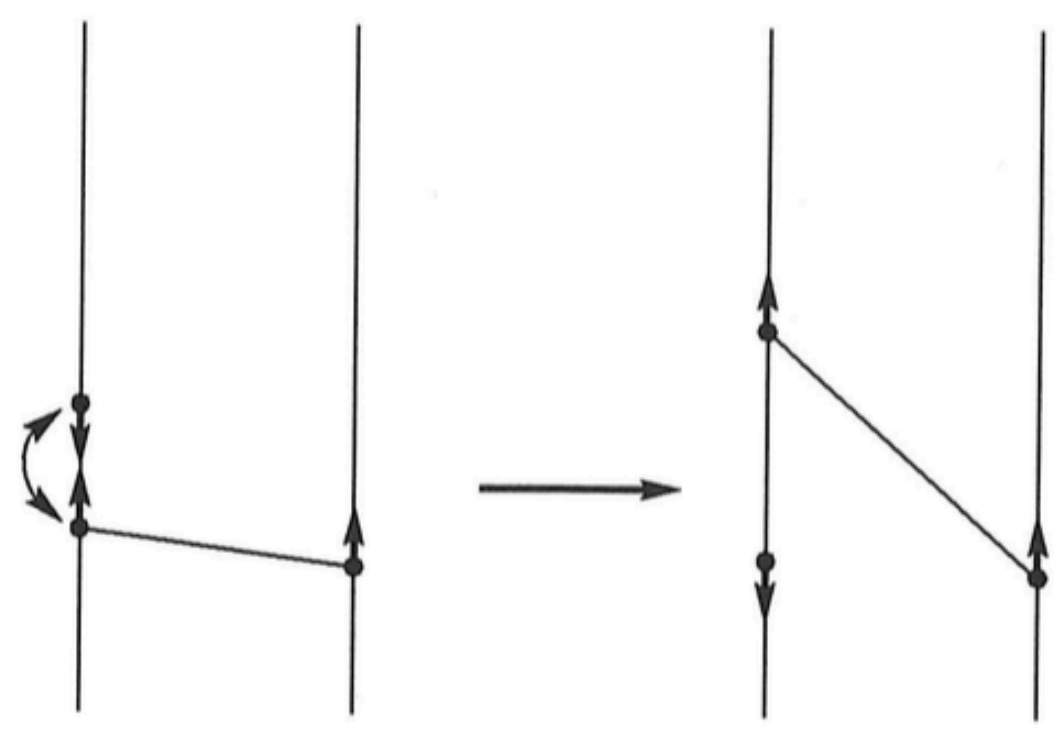

FIGURE 5

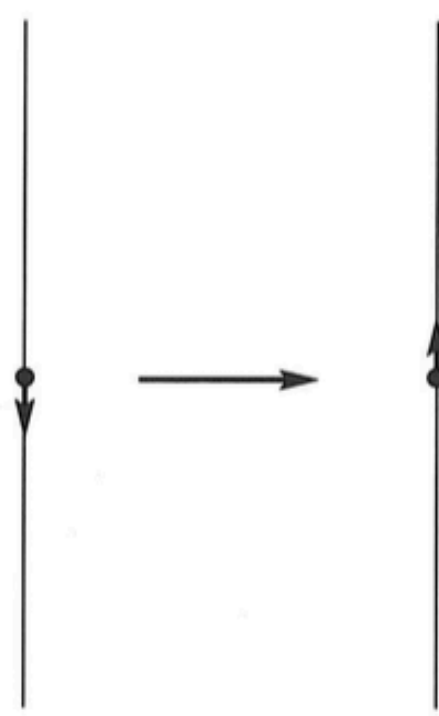

$\lambda$

$\lambda$

Figure 6

interior critical points of the function, then the abstract FMC corresponding to the final sphere is obtained from the abstract FMC corresponding to the initial sphere by the composition of some metamorphoses of types 1-3.

Proof. This follows directly from general theorems describing the metamorphoses of the Morse complex in a 1-parameter family (see, e.g., [1, 6]).

REMARK 1. The metamorphoses of the framed Morse complex of a function in indices $0,1,(n-2),(n-1)$ induce very special cases of metamorphoses of the abstract FMC.

Consider the transformation of an abstract FMC when the sphere to which we restrict the function moves through an interior index $\lambda$ critical point of the function.

Lemma 1. A family of spheres $S_{t}, t \in[-\varepsilon, \varepsilon]$, intersecting at $t=0$ an interior index $\lambda$ critical point of the function and coinciding outside a neighborhood of the critical point, can be chosen in such way that the abstract FMC 
corresponding to the final sphere $S_{\varepsilon}$ is obtained from that corresponding to the initial sphere $S_{-\varepsilon}$ by a type 4 metamorphosis of index $\lambda$.

Proof. One can choose coordinates $x_{1}, \ldots, x_{n}$ in a neighborhood $U$ of the critical point so that

$$
f=-\sum_{i=1}^{\lambda} x_{i}^{2}+\sum_{i=\lambda+1}^{n} x_{i}^{2}+c
$$

in this neighborhood and that the metric in this neighborhood is symmetric with respect to the hyperplane $x_{i}=0$ for some $i$ (after an appropriate change of the metric in $U$, if necessary). Define the family of spheres so that the following conditions are satisfied:

(1) outside $U$ all spheres coincide;

(2) in some smaller neighborhood $V$ of the critical point the family is defined by the equation $x_{i}=c, c \in[-\varepsilon, \varepsilon]$;

(3) the intersections of the spheres $S_{\varepsilon}$ and $S_{-\varepsilon}$ with $U$ are symmetric with respect to the hyperplane $x_{i}=0$ and the restriction of the function to these spheres has no critical points in $U \backslash V$.

Then the restrictions of the function and of the metric to the spheres $S_{\varepsilon}$ and $S_{-\varepsilon}$ coincide. The framed Morse complexes for these spheres differ only by the directions of the gradient at the critical point in $U$. Hence $S_{t}$ is the required family of spheres.

1.4. A necessary condition for the existence of a smooth extension of a function to the interior of the ball with exactly $n$ interior nondegenerate critical points. Consider a linear function on the standard $n$-dimensional ball in $\mathbb{R}^{n}$. The abstract FMC of such a function is shown in Figure 7.

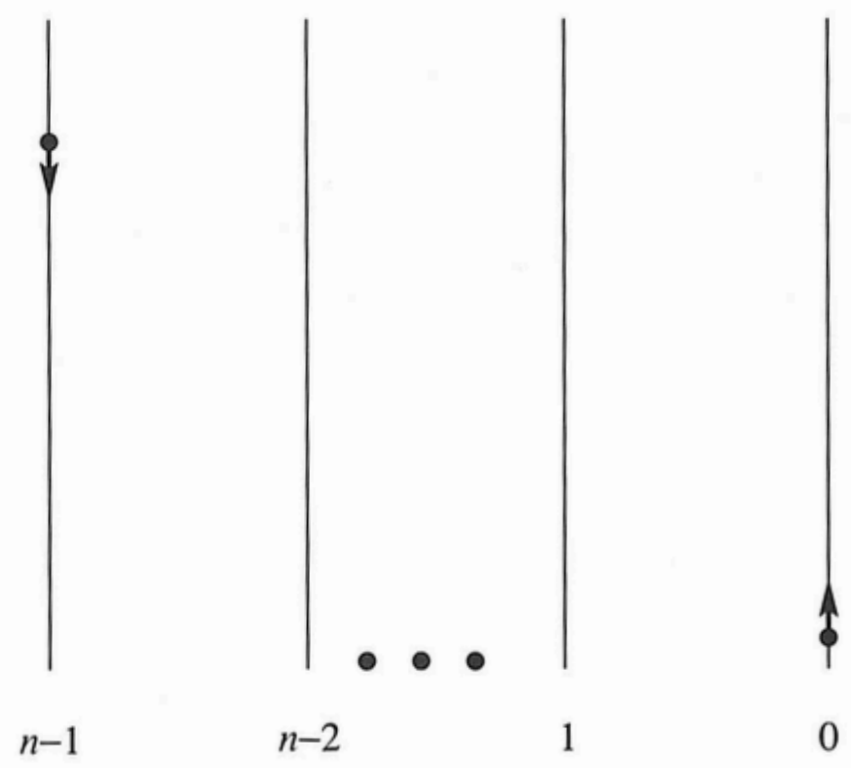

FIGURE 7. The abstract FMC of a linear function

THEOREM 2. If a function on a neighborhood of the boundary of an ndimensional ball can be extended smoothly inside the ball to a function with 
$n$ critical points of indices $\lambda_{1}, \ldots, \lambda_{n}$, then the abstract FMC corresponding to the given function can be reduced to the simplest abstract FMC shown in Figure 7 by the composition of certain metamorphoses of types 1-3 and n metamorphoses of type 4 of the corresponding indices.

PROof. Consider a sphere bounding a small neighborhood of a noncritical point in the ball. The abstract FMC for the restriction of the function to this sphere is the simplest one (see Figure 7). Now the result follows directly from Theorem 1 and Lemma 1 applied to a family connecting the two spheres.

REMARK 2. Taking into account the subtleties in the metamorphoses in indices $0,1,(n-2),(n-1)$ (see Remark 1$)$ it seems likely (see, e.g., $[1,3,6]$ ) that for large $n$ this condition should be sufficient. But there is one obstacle for the realization of the complex metamorphoses 3 for a given function. There might be a family of gradient curves connecting two critical points of $\left.f\right|_{S^{n-1}}$ of indices, for example, $\lambda+2$ and $\lambda$, which has no boundary. This family would not be reflected in the abstract FMC. However, such a family provides an obstruction for the increase of the critical value of index $\lambda$.

Remark 3. Similarly, the study of the framed Morse complex provides a necessary condition for the existence of a smooth continuation of a function to the interior of the ball with one (two, three, ... etc.), possibly degenerate, critical points. One can vary slightly the continuation of the function inside the ball in such way that the continuation of the function will have some nondegenerate critical points with neighboring critical values instead of the degenerate critical points. Hence the abstract FMC of the given function is reducible to the simplest abstract FMC shown in Figure 7 by composition of some arbitrary metamorphoses of types 1-3 and certain metamorphoses of type 4 on one (two, three, ..., etc.) levels.

\section{§2. Invariants of the framed Morse complex and a lower bound for the number of critical points}

\subsection{Definition of the invariants.}

Definition. The chain complex $C_{*}$ with fixed generators in each $C_{k}$ is of the simplest form if for every $k$ and every generator $a$ in $C_{k}$ its boundary $d a$ is either zero or a generator in $C_{k-1}$ provided that the boundaries of different generators are different.

For example, the abstract FMC shown in Figure 8 is of the simplest form.

Definition. A complex $C_{*}$ is said to be a complex with ordered generators if in each of its chain groups $C_{k}$ an ordered set of generators is fixed. We regard two such complexes as equivalent if their chain groups have the same dimensions and the differentials on corresponding generators coincide.

Consider the chain complex of an abstract FMC. There is a natural order for the generators of each chain group $C_{k}$. It is defined by the order of the corresponding heights. Let $e_{i}^{j}$ denote the generator in $C_{j}$ corresponding to the $i$ th height in increasing order among the heights of index $j$. Consider the complex $C_{*} \otimes F$ over some field $F\left(F=\mathbb{Q}, \mathbb{Z}_{p}\right)$. 


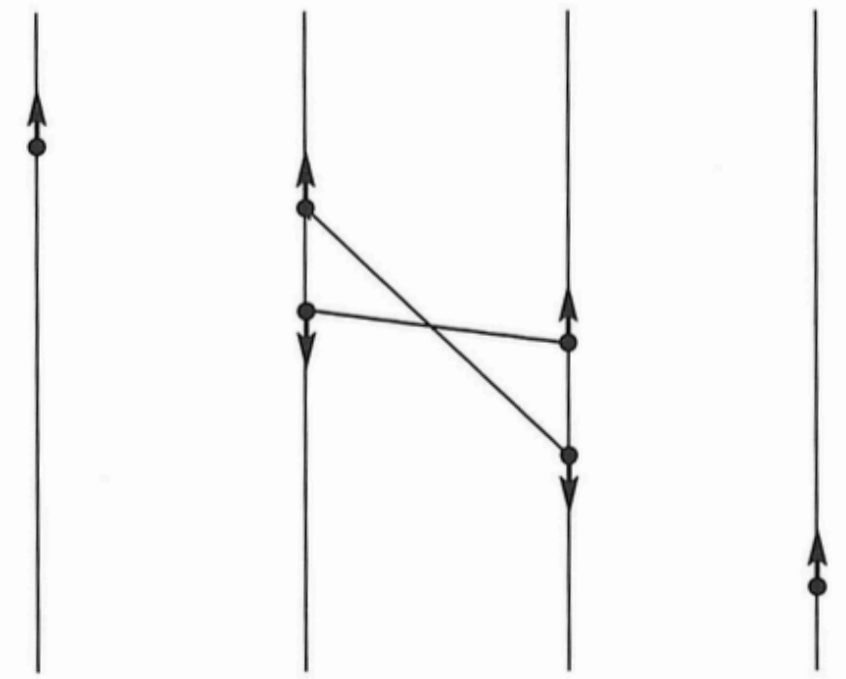

FigURE 8. An abstract FMC having the simplest form

LeMma 2. Let the chain complex $C_{*}$ be a complex with ordered generators. Then it can be reduced over $F$ to a complex with ordered generators of simplest form by upper-triangular matrices $A_{j} \in \operatorname{Aut}\left(C_{j}\right)$ :

$$
A_{j}=\left(\begin{array}{cccc}
f_{11} & f_{12} & \ldots & f_{1 k} \\
0 & f_{22} & & \vdots \\
\vdots & \ddots & \ddots & \vdots \\
0 & 0 & \ldots & f_{k k}
\end{array}\right), \quad f_{m n} \in F .
$$

This complex with ordered generators of simplest form is uniquely defined by the original complex.

Definition. We shall call this complex of simplest form the canonical form over $F$ of the given complex with ordered generators.

Proof OF LemMa 2. Essentially it follows from the fact that

$$
G L(n, F) /(T(n, F) \times T(n, F))=S(n),
$$

where $T(n, F)$ is the group of upper-triangular matrixes, $S(n)$ is the permutation group, and $T(n, F) \times T(n, F)$ acts on $G(n, F)$ by changes of bases in image and preimage (see [1]). The complete proof is presented below. Suppose that for $p=j$ and $m \leq i$, or $p<j$ and all $m, d e_{m}^{p}$ has the required form, i.e., either $d e_{m}^{p}=e_{k(m)}^{p-1}$ or $d e_{m}^{p}=0$. We shall reduce $d e_{i+1}^{j}$. Let $d e_{i+1}^{j}=\sum e_{k}^{j-1} \alpha_{k}, \alpha_{k} \in F$. Let us move all the terms for $e_{k}^{j-1}=d e_{q}^{j}$ with $q \leq i$ from the right-hand side to the left-hand side. We get

$$
d\left(e_{i+1}^{j}-\sum_{q=1}^{i} e_{q}^{j} \alpha_{k(q)}\right)=\sum_{k} e_{k}^{j-1} \beta_{k}
$$

If $\beta_{k}=0$ for any $k$, then define $\tilde{e}_{i+1}^{j}=e_{i+1}^{j}-\sum_{q=1}^{i} e_{q}^{j} \alpha_{k(q)}$. Otherwise, 
there is $k_{0}$ such that

$$
d\left(e_{i+1}^{j}-\sum_{q=1}^{i} e_{q}^{j} \alpha_{k(q)}\right)=e_{k_{0}}^{j-1} \beta_{k_{0}}+\sum_{k<k_{0}} e_{k}^{j-1} \beta_{k}, \quad \beta_{k_{0}} \neq 0,
$$

where $k_{0} \neq k(q)$ for every $q \leq i$ and $d e_{k}^{j-1}=0$ for every $k$ with $\beta_{k} \neq 0$. Define

$$
\tilde{e}_{i+1}^{j}=\left(e_{i+1}^{j}-\sum_{q=1}^{i} e_{q}^{j} \alpha_{k(q)}\right) / \beta_{k_{0}}, \quad \tilde{e}_{k_{0}}^{j-1}=e_{k_{0}}^{j-1}+\sum_{k<k_{0}} e_{k}^{j-1} \beta_{k} / \beta_{k_{0}} \cdot,,
$$

Then the following two facts hold. First, $d \tilde{e}_{i+1}^{j}=\tilde{e}_{k_{0}}^{j-1}$. Second, for $p<j$ and all $m$ or $p=j$ and $m \leq i$ the expression $d e_{m}^{p}$ continue to have the required form. If the complex has been reduced to canonical form up to the index $j$, then one can similarly reduce $d e_{1}^{j+1}$ and so on.

Let us prove the uniqueness of the canonical form over $F$ of the complex $C_{*}$. Assume that there are two different canonical forms. Let $\left\{a_{i}^{j}\right\}$ and $\left\{b_{i}^{j}\right\}$ be the ordered generators of $C_{j}$ for these canonical forms. Assume that for $p<j$ and any $m$, or for $p=j$ and $m<i$ the canonical forms coincide, i.e. if $d a_{m}^{p}=a_{k}^{p-1}$, then $d b_{m}^{p}=b_{k}^{p-1}$. Let $d a_{i}^{j}=a_{m}^{j-1}$ and $d b_{i}^{j}=b_{l}^{j-1}$ with $m>l$. Note that

$$
b_{i}^{j}=\sum_{k=1}^{i} a_{k}^{j} \alpha_{k}, \quad b_{l}^{j-1}=\sum_{n=1}^{l} a_{n}^{j-1} \beta_{n},
$$

where $\alpha_{k}, \beta_{n} \in F$ and $\alpha_{i}, \beta_{l} \neq 0$. Hence

$$
d\left(\sum_{k=1}^{i} a_{k}^{j} \alpha_{k}\right)=\sum_{n=1}^{l} a_{n}^{j-1} \beta_{n} .
$$

Therefore

$$
d a_{i}^{j}=\sum_{n=1}^{l} a_{n}^{j-1} \beta_{n} / \alpha_{i}-\sum_{k=1}^{i-1} d a_{k}^{j} \alpha_{k} / \alpha_{i} .
$$

On the other hand, $d a_{i}^{j}=a_{m}^{j-1}, m>l, a_{m}^{j-1} \neq d a_{k}^{j}$ for any $k \neq i$. This proves the uniqueness of the canonical form of $C_{*}$ over $F$.

DEFINITION. If our chain complex is the chain complex of an abstract FMC, then its canonical form over $F$ is the chain complex of a unique abstract FMC. This abstract FMC is called the canonical form over $F$ of the original abstract FMC.

EXAMPLE. See Figure 9.

LeMMA 3. The canonical form of an abstract FMC corresponding to a generic smooth function does not depend on the metric.

Proof. Consider two abstract FMC's defined by the same function in different metrics. Connect the two metrics by a generic family. Then the 


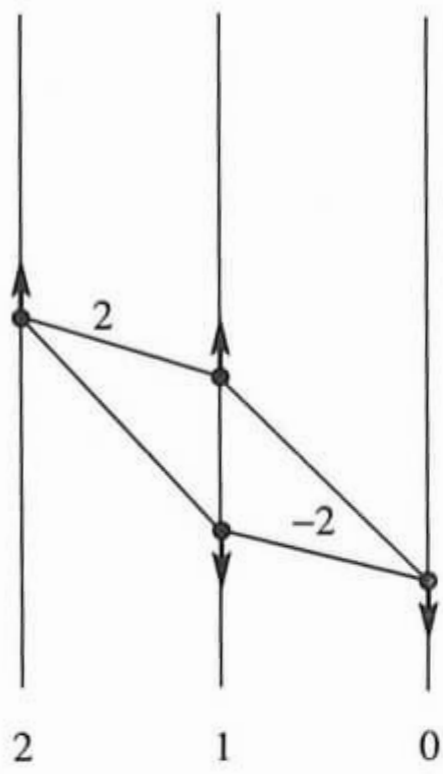

An abstract FMC

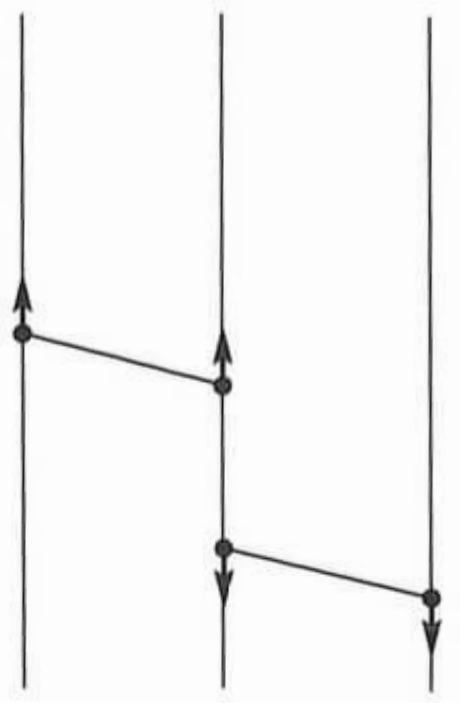

2

Its canonical form over $\mathbb{Q}$

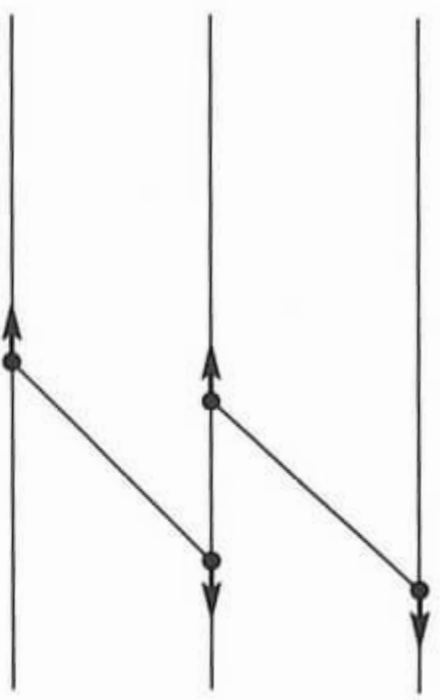

2

1

0

Its canonical form over $\mathbb{Z}_{2}$

\section{FIGURE 9}

abstract FMC corresponding to the final metric is obtained by composition of some metamorphoses of type 1 from the one corresponding to the initial metric. Hence one of the given complexes is reduced to the other by applying several substitutions of the bases in all $C_{k}$ of the form

$$
A_{k}=\left(\begin{array}{cccc}
1 & \ldots & \ldots & a_{1 l} \\
0 & 1 & & \vdots \\
\vdots & \ddots & \ddots & \vdots \\
0 & 0 & \ldots & 1
\end{array}\right), \quad a_{i j} \in \mathbb{Z}
$$

Therefore the canonical forms of such abstract framed Morse complexes coincide over an arbitrary field.

REMARK 1. There is a natural complete flag defined by the order of the generators in each $C_{k} \otimes F$ for any abstract FMC, which is not changed by the transformations of bases in metamorphosis 1 . The canonical form of the abstract FMC reflects the relation between the two structures (chain complex and the flags) of the abstract FMC.

The canonical forms over $\mathbb{Q}$ and $\mathbb{Z}_{p}$ of the abstract FMC's are the invariants we are going to study.

2.2. Metamorphoses of the canonical forms. In what follows we shall consider an abstract FMC having the same homology as $S^{n-1}$.

LEMMA 4. The standard metamorphoses of an abstract FMC act on its canonical form in the following way:

(1) The canonical form does not change under metamorphoses of type 1.

(2) A pair of heights appears or disappears under a metamorphosis of type 2 in the canonical form, as in the abstract FMC (such a pair is always connected 
by a line segment in the canonical form).

(3) A height is shifted in the canonical form, as in the abstract FMC, under the metamorphosis 3a. Under the metamorphosis $3 \mathrm{~b}$ the canonical form is transformed in one of the 9 ways shown on Figure 10. Since the canonical form of the corresponding complexes does not depend on the directions of the heights we do not show them and show only which of the heights are exchanged. We show a part of the canonical form. The rest does not change.

Which version of the metamorphoses 3.1 and 3.2 is actually carried out, is determined by the original abstract FMC.

In metamorphoses 3.1 and 3.2 we can have $\lambda=n-1$ or $\lambda=0$. In these cases one must simply delete the heights of indices $\lambda=n$ or $\lambda=-1$ from the figures.

(4) The direction of a height is switched under a metamorphosis of type 4 (as in an abstract FMC).

Proof. In cases 1, 2, 3a, 4 the proof is obvious.

Consider case $3 \mathrm{~b}$. Let us prove that it is sufficient to consider the case of a complex consisting of 4 heights: two exchanging heights and two heights connected with them (see Figure 10). Denote by $\left\{e_{i}^{j}\right\}$ and $\left\{g_{i}^{j}\right\}$ the ordered generators in spaces $C_{j} \otimes F$ corresponding to the given abstract FMC and its canonical form (see $\S 2.1$ ). Let the exchanged heights have index $p$ and numbers $q$ and $q+1$ in increasing order. Let $\left\{\bar{e}_{i}^{j}\right\}$ denote the ordered generators corresponding to the abstract FMC after the metamorphosis. Then

$$
g_{q+1}^{p}=\alpha_{q+1} e_{q+1}^{p}+\alpha_{q} g_{q}^{p}+\sum_{i<q} \alpha_{i} g_{i}^{p}
$$

Define

$$
\bar{g}_{i}^{j}=g_{i}^{j} \text { for } j \neq p \text { or } j=p \text { and } i \neq q+1, \bar{g}_{q+1}^{p}=\alpha_{q+1} e_{q+1}^{p}+\sum_{i<q} \alpha_{i} g_{i}^{p} \text {. }
$$

Then the bases $\left\{\bar{g}_{i}^{j}\right\}$ are obtained from the bases $\left\{\bar{e}_{i}^{j}\right\}$ by upper-triangular substitutions. Also the bases $\left\{\bar{g}_{i}^{j}\right\}$ are obtained from the bases $\left\{e_{i}^{j}\right\}$ by upper-triangular substitutions. In the bases $\left\{\bar{g}_{i}^{j}\right\}$ the complex is reduced to its canonical form except for possibly the subcomplex corresponding to the above-mentioned 4 heights.

Now consider a complex consisting of 4 heights (see Figure 10). Consider the case 3.1 (Figure 11). In this case $f_{b}^{a} \neq 0, f_{d}^{c} \neq 0$. If $f_{c}^{a} \neq 0$, then version 3.1 .1 occurs. Otherwise, $f_{c}^{a}=0, f_{d}^{b}=0$ and version 3.1 .2 occurs. Other cases 3.2-3.4 are similar.

The lemma is proved.

REMARK 2. The assumption that the homology of the abstract FMC under consideration coincides with the homology of $S^{n-1}$ is made only for simplicity.

REMARK 3. Since the metamorphoses 3 a do not change the canonical form in an essential way we shall not mention them unless necessary. 
3.1

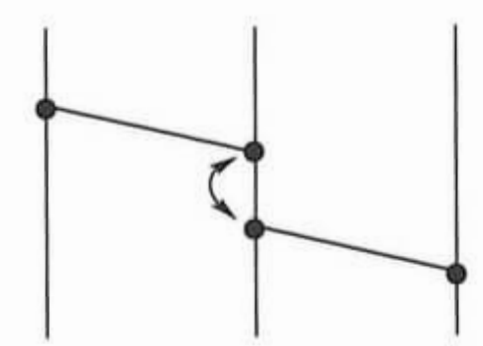

$\lambda+1$ $r^{3.1 .2}$
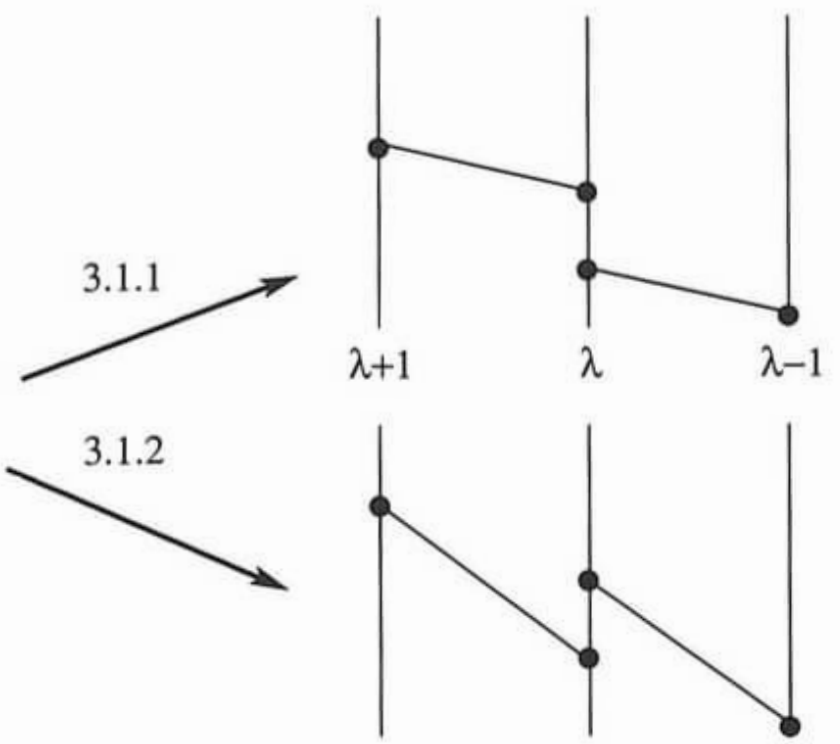

3.2
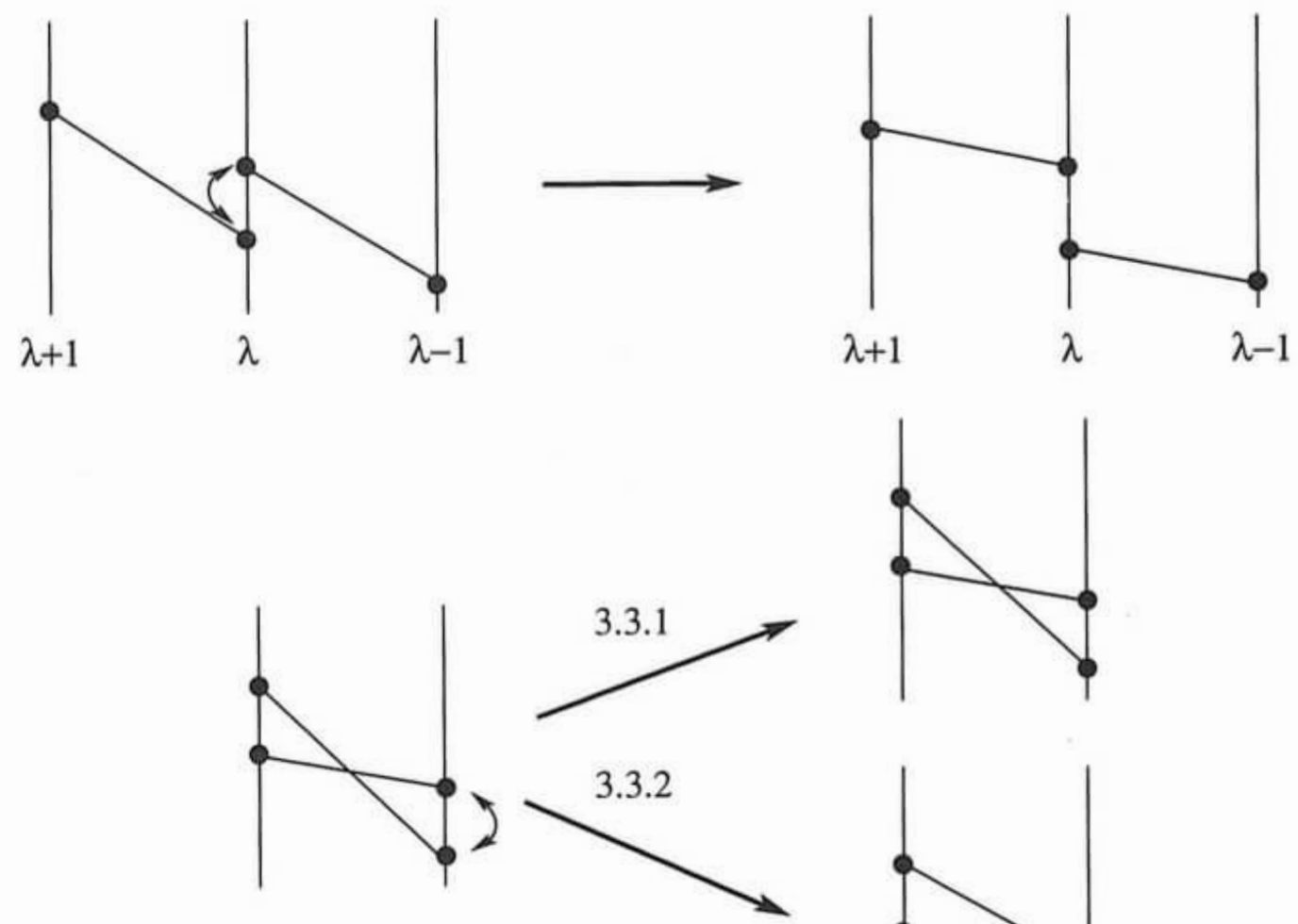

3.3
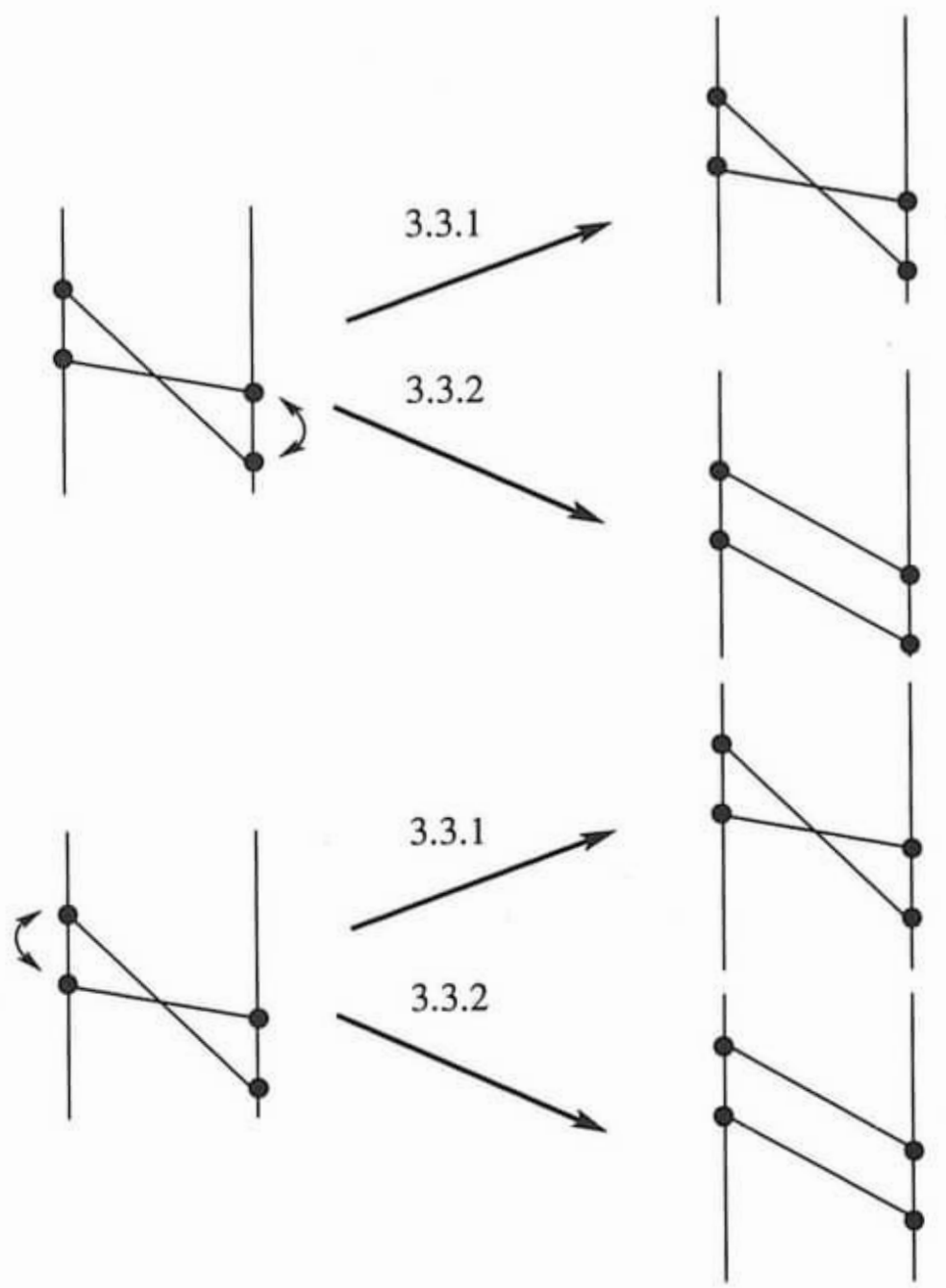

FIGURE 10 

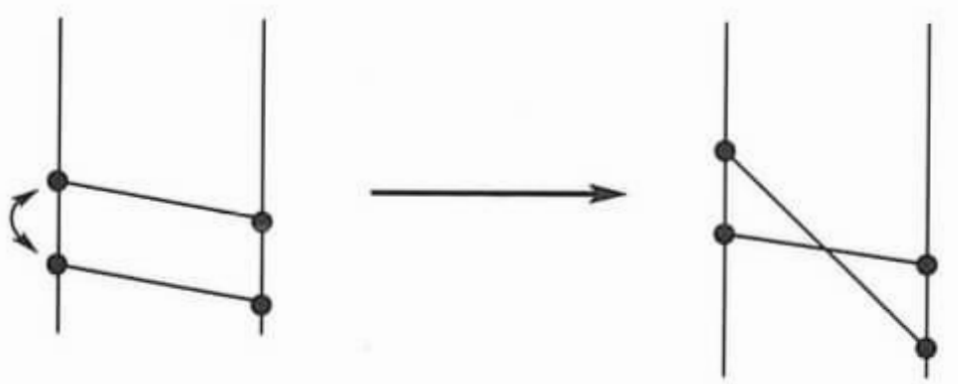

3.4
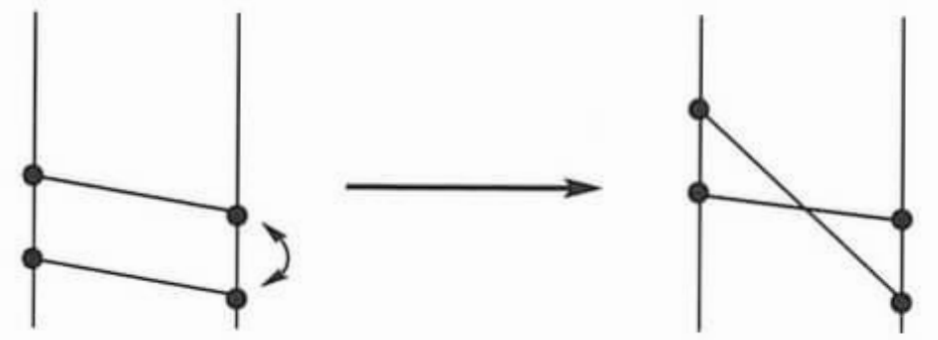

FIGURE 10 (continued)

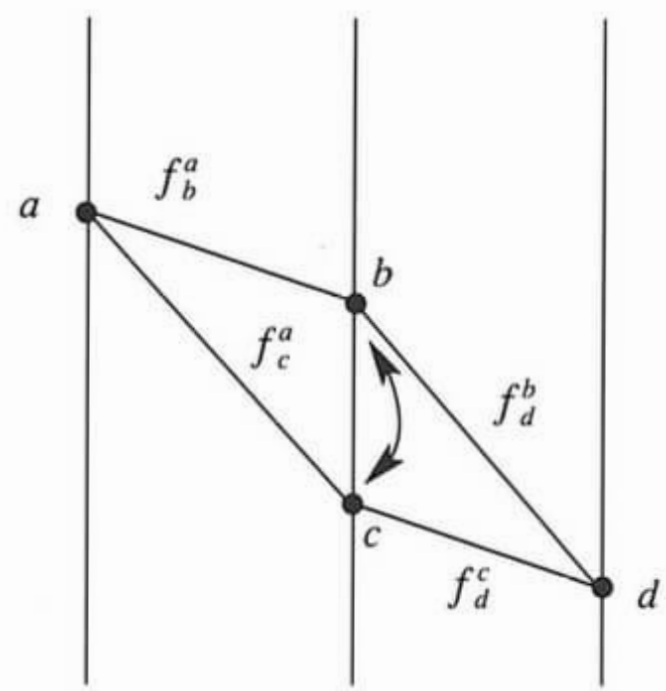

FIGURE 11

Consider the types of pairs of heights connected by a line segment in the canonical form. There are two types of pairs of heights with different directions. They are shown in Figure 12.

2.3. Metamorphoses of type 3 that change the number of pairs of types 1 and 2.

Lemma 5. Metamorphosis of the types 3.1-3.4 of canonical form changes the numbers of pairs of type 1 and 2 only in the following cases (Figure 13-17):

(1) reduction of a pair of type 1 and a pair of type 2. (Figure 13)

(2) reduction of a pair of type 1 and a pair of type 2. (Figure 14)

(3) metamorphosis of a pair of type 1 to a pair of type 2. (Figure 15)

(4) birth of a pair of type 1 and a pair of type 2 . (Figure 16) 


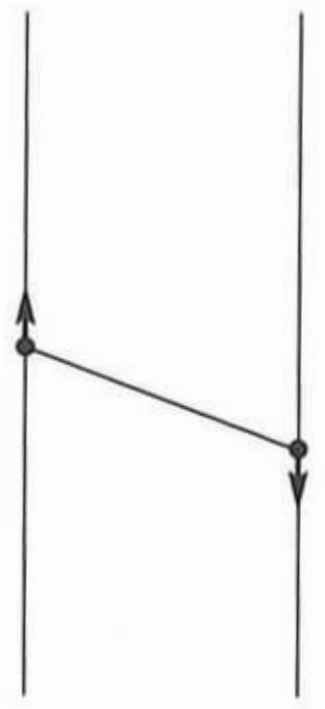

1.

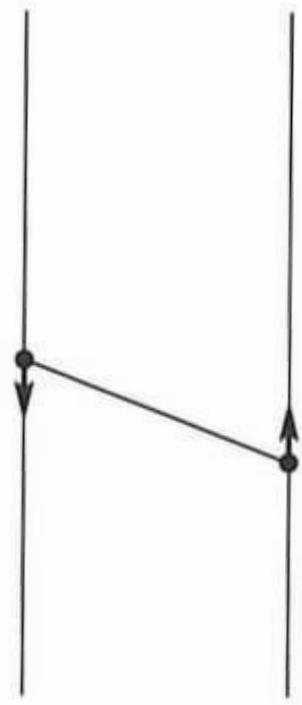

2.

FIGURE 12. Types of heights with different directions connected by a line segments
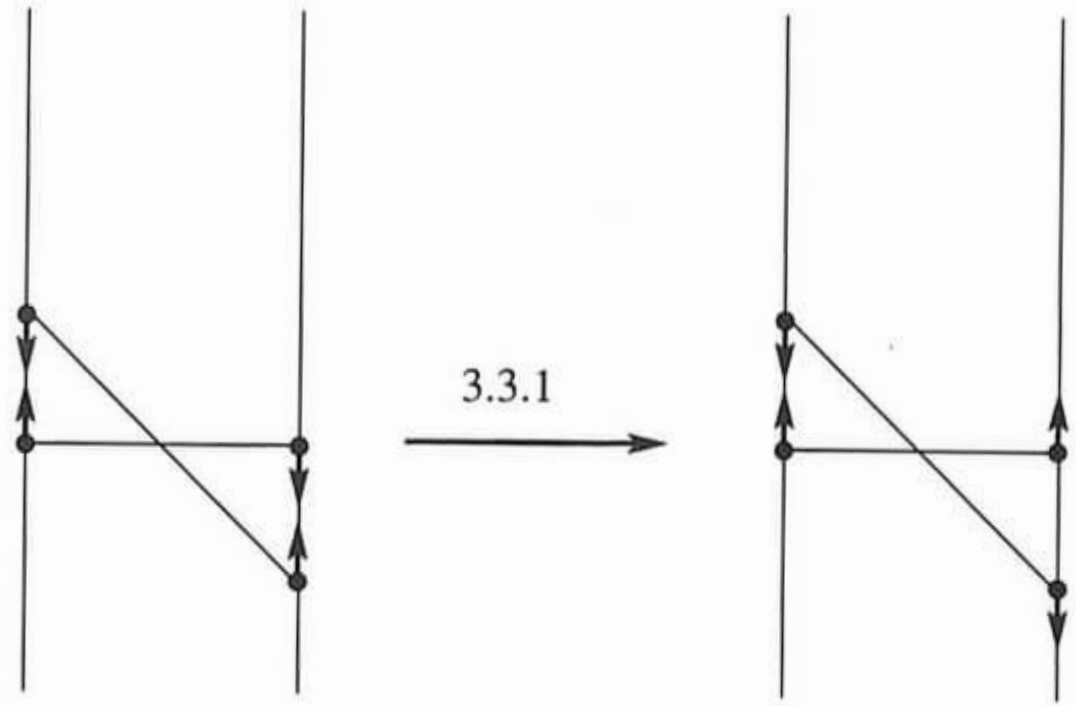

FIGURE 13

(5) birth of a pair of type 2 and a pair of type 2. (Figure 17)

REMARK 4. There are also metamorphoses involving a pair of heights connected by a segment and a height $\tilde{a}$ representing a nonzero homology class that change the numbers of pairs of types 1 and 2 . All such possible metamorphoses are: metamorphosis 5 with $\lambda=n-1$ or $\lambda=0$ and metamorphosis 3 with $\lambda=0$ in the first case and $\lambda=n-1$ in the second case. It is convenient to regard the height $\tilde{a}$ as a pair of type 2 if it has index $\lambda=n-1$ and direction "up" or index $\lambda=0$ and direction "down". Then metamorphoses 3 and 5 keep their names in cases $\lambda=0$ and $\lambda=n-1$.

PROOF. Lemma 5 follows directly from Lemma 4.

2.4. Optimal sequence of metamorphoses of the canonical form. In what follows we consider metamorphoses of canonical form of an abstract FMC 

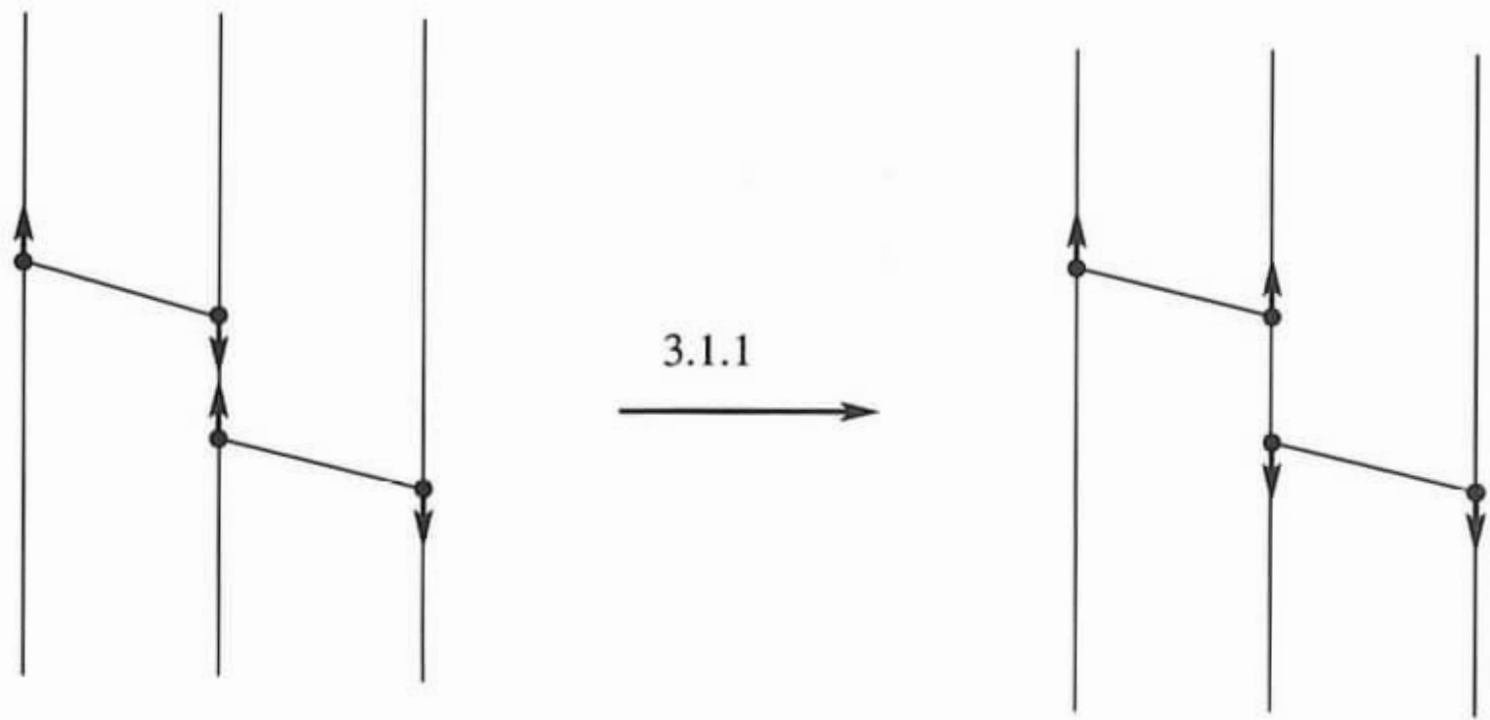

Figure 14

abstracting from the metamorphoses of the abstract FMC. In other words we consider arbitrary metamorphoses of types 2, 3.1-3.4, 4 (see Lemma 4) of an abstract FMC having the simplest form (see §2.1).

Consider all sequences of metamorphoses 2, 3.1-3.4, 4 of the given canonical form by which this canonical form can be reduced to the simplest abstract FMC shown in Figure 7. Our aim is to calculate the minimal number of metamorphoses of type 4 in such a sequence for the given canonical form. We shall call a sequence involving the minimal number of metamorphoses of type 4 an optimal sequence.

By Theorem 2, a solution of this problem provides a lower bound for the number of critical points of continuations of the given function to the interior of the ball.

If an optimal sequence of metamorphoses of the canonical form is induced by a sequence of metamorphoses of an original abstract FMC, then this sequence of metamorphoses of the abstract FMC is optimal in a similar sense. However simple examples show that there exist metamorphoses of types 2, 3.1, 3.3 of a canonical form that cannot be induced by metamorphoses of the corresponding abstract FMC.

LEMMa 6. Any sequence of metamorphoses of types 2, 3.1-3.4, 4 of canonical form can be replaced by a sequence such that

(1) all metamorphoses of type 4 appear in this sequence after all metamorphoses 3 that change numbers of pairs of types 1 and 2;

(2) the set of indices of metamorphoses 4 appearing in this sequence is the same as the one for the given sequence;

(3) the new sequence yields the same final canonical form.

Proof. Consider any type 4 metamorphosis in this sequence. Suppose that there exists a height connected by a line segment with the height that switches direction. After the metamorphosis these heights have either 

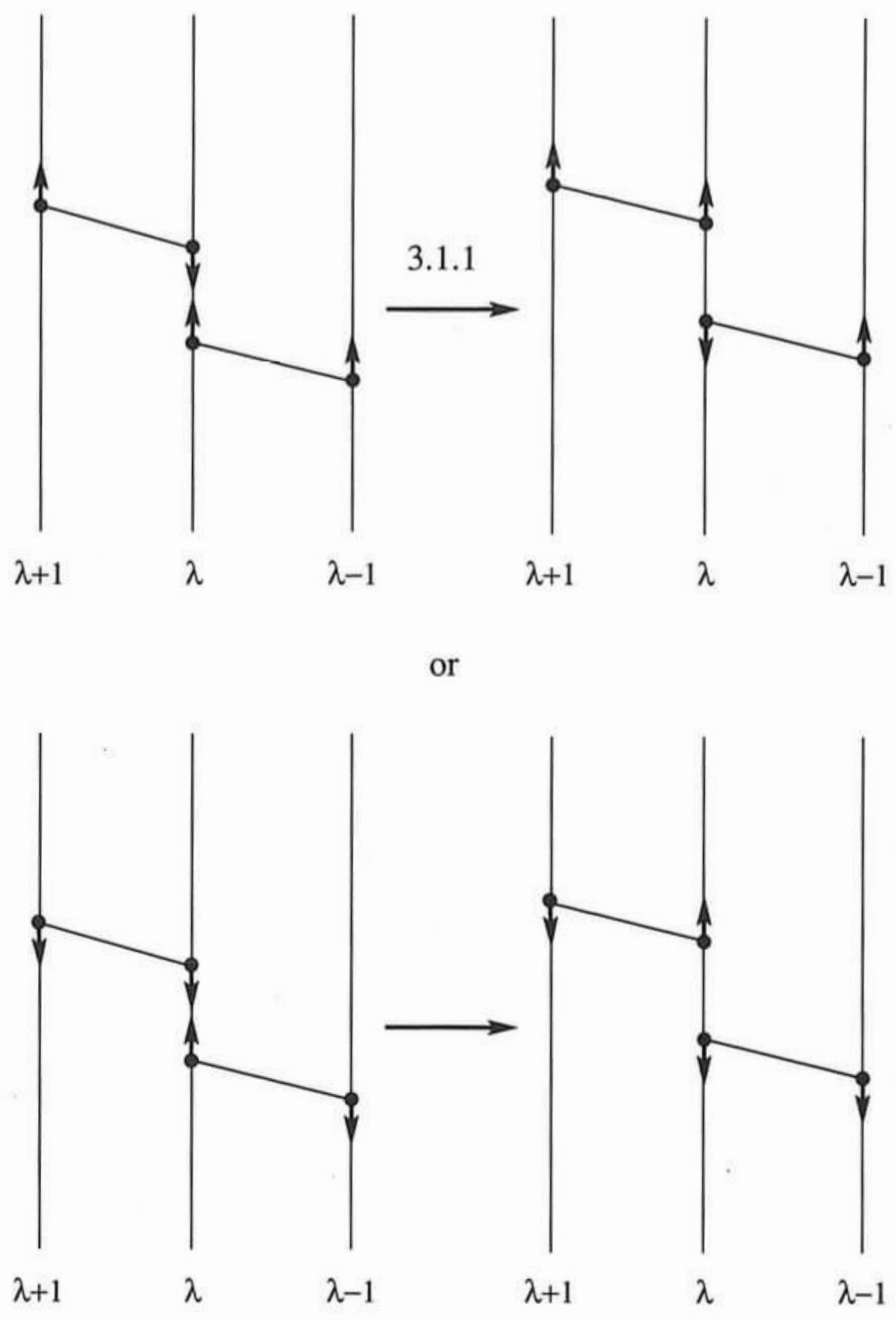

FIGURE 15

identical or different directions. In the first case we replace the metamorphosis 4 by a metamorphosis $2 a$ that gives the same pair of heights and the same metamorphosis 4 disposed after all metamorphoses 3.1-3.4 together with that metamorphosis $2 \mathrm{~b}$ destroying the pair of heights that appeared after the metamorphosis 4 (Figure 18). In the second case we replace the metamorphosis 4 by the composition of metamorphoses of types $2,3.1,3.3$ that gives the same pair of heights and another pair of heights with different directions according to the case 4 in Lemma 5, and the metamorphosis 4 together with a metamorphosis $2 \mathrm{~b}$ placed after all metamorphoses 3.1-3.4 and destroying the second pair (Figure 19). The case when the height that switches direction represents a nonzero homology class is similar.

THEOREM 3. For any canonical form there exists an optimal sequence of its 


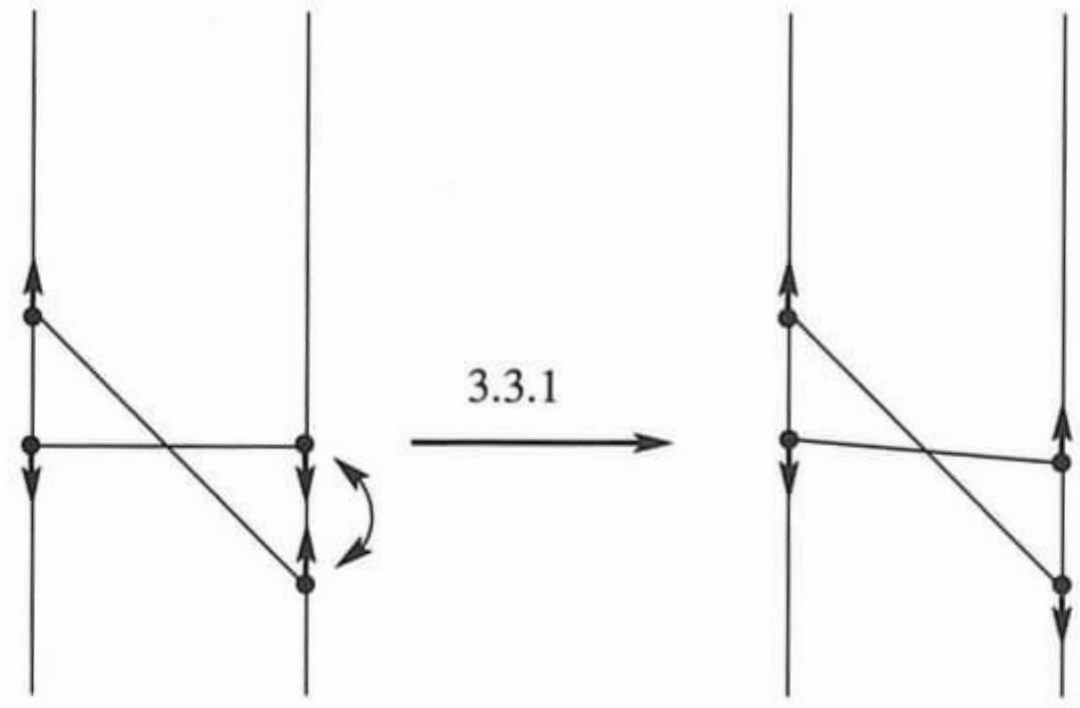

FIGURe 16

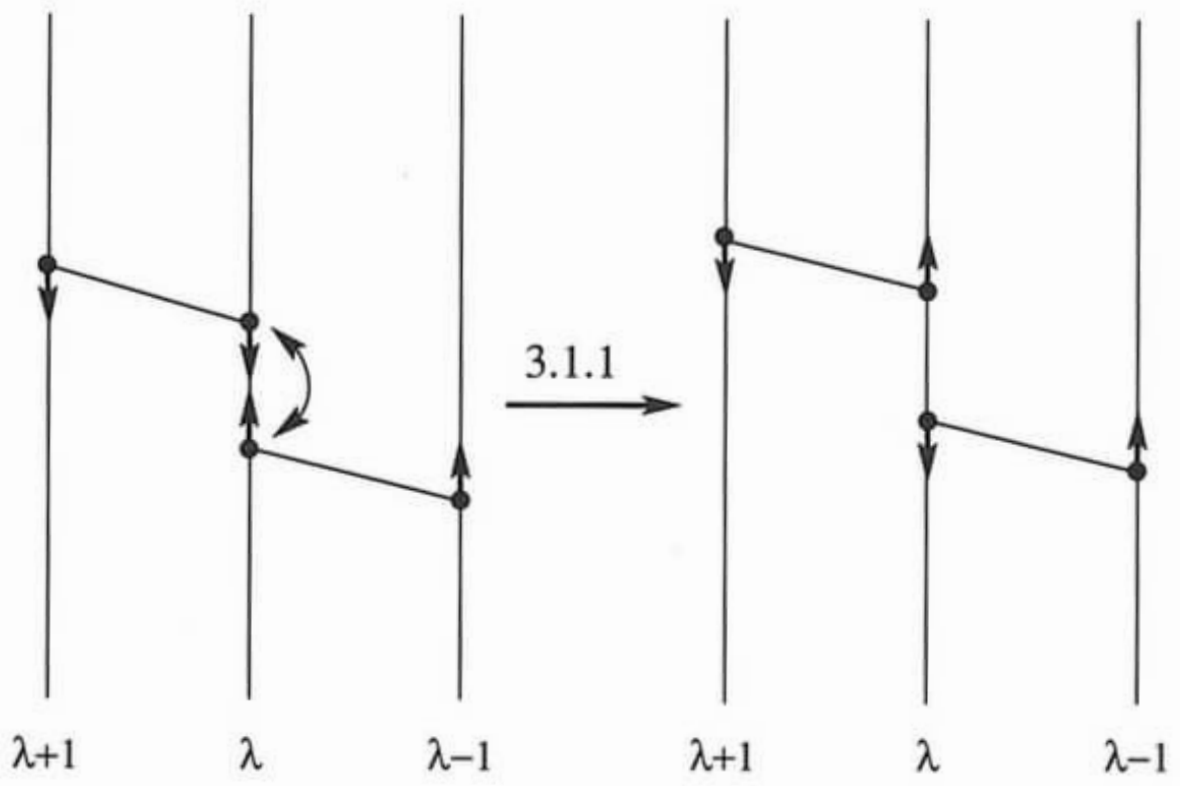

FIGURE 17

metamorphoses containing no metamorphoses 2a of births of pairs of heights.

COROLLARY. An optimal sequence for any canonical form can be found by looking through finite number of sequences of reductions of pairs of type 1 and 2.

Proof of Theorem 3. By Lemma 6, there exists an optimal sequence of metamorphoses of the given canonical form with all metamorphoses 4 occuring after all metamorphoses 3.1-3.4 that change the numbers of pairs of types 1 and 2. Consider the sequence of metamorphoses up to the first metamorphosis 4. This subsequence of metamorphoses of types 2, 3.1-3.4 transforms the given canonical form into a canonical form having a minimal number of pairs of types 1 and 2 among all sequences of metamorphoses of types 2, 3.1-3.4. We shall show that some metamorphoses in this sequence can be replaced in such way that this sequence will not include any metamorphosis $2 \mathrm{a}$ and will yield the same final total number of pairs of types 1 and 2 . 


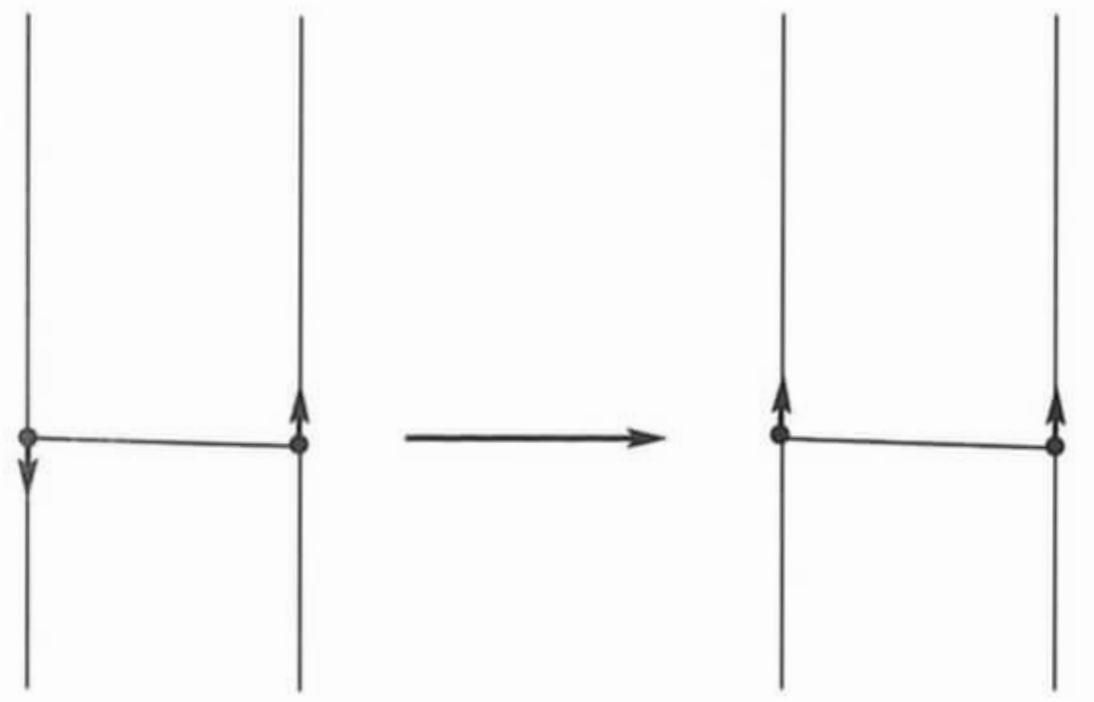

We replace by

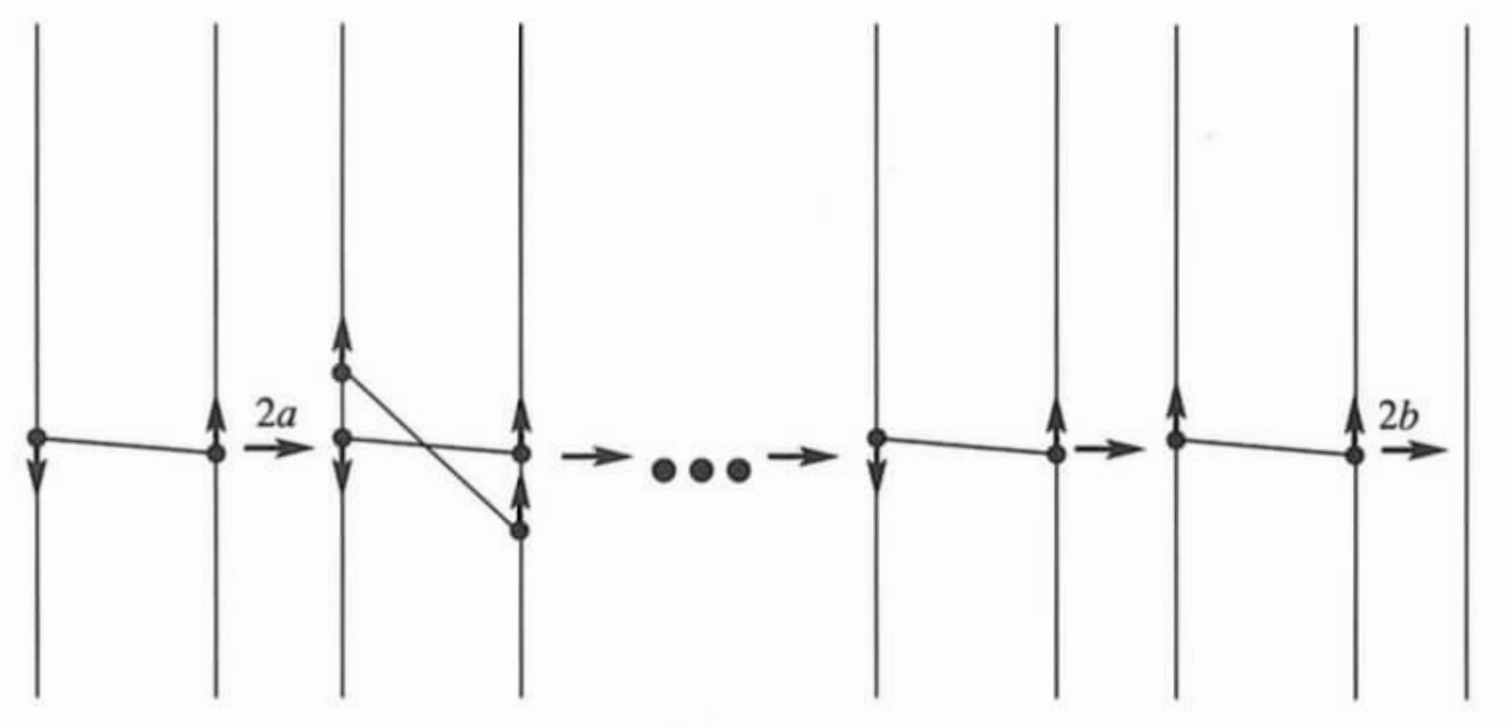

FIGURE 18

A pair of heights with different directions connected by a segment is reduced in the given sequence together that another such pair which either comes from the initial canonical form or appears together with another such pair according to Lemma 5. In the last case the total number of pairs of types 1 and 2 does not change. But the remaining pair can have a more useful position for annihilation with another pair. We shall show that, conversely, the remaining pair can be obtained by a simple shift of heights from the initial pair. A similar result holds for metamorphosis of a pair of type 1 to a pair of type 2. Thus we can replace all metamorphoses in which a pair of type 1 or 2 is born and then is reduced by metamorphoses containing no metamorphoses $2 \mathrm{a}$. 


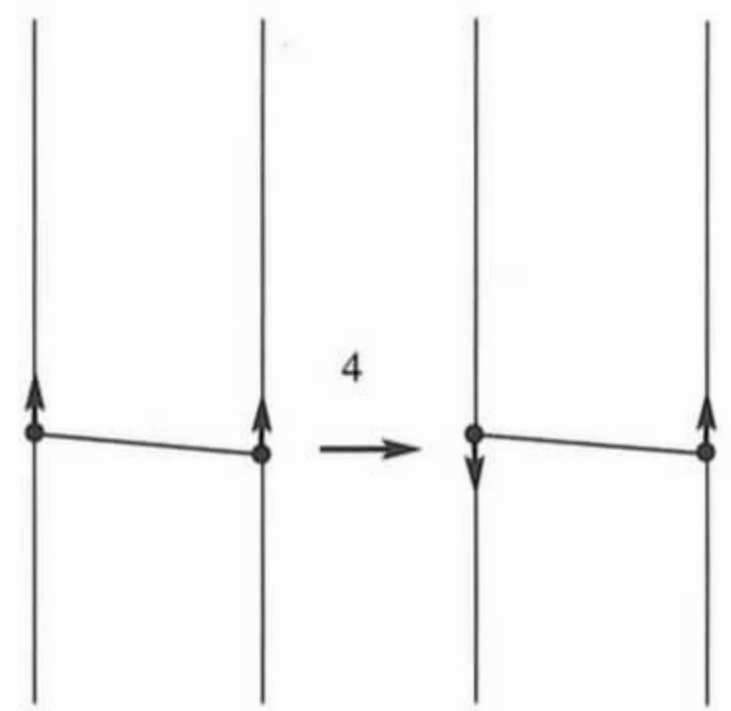

We replace by

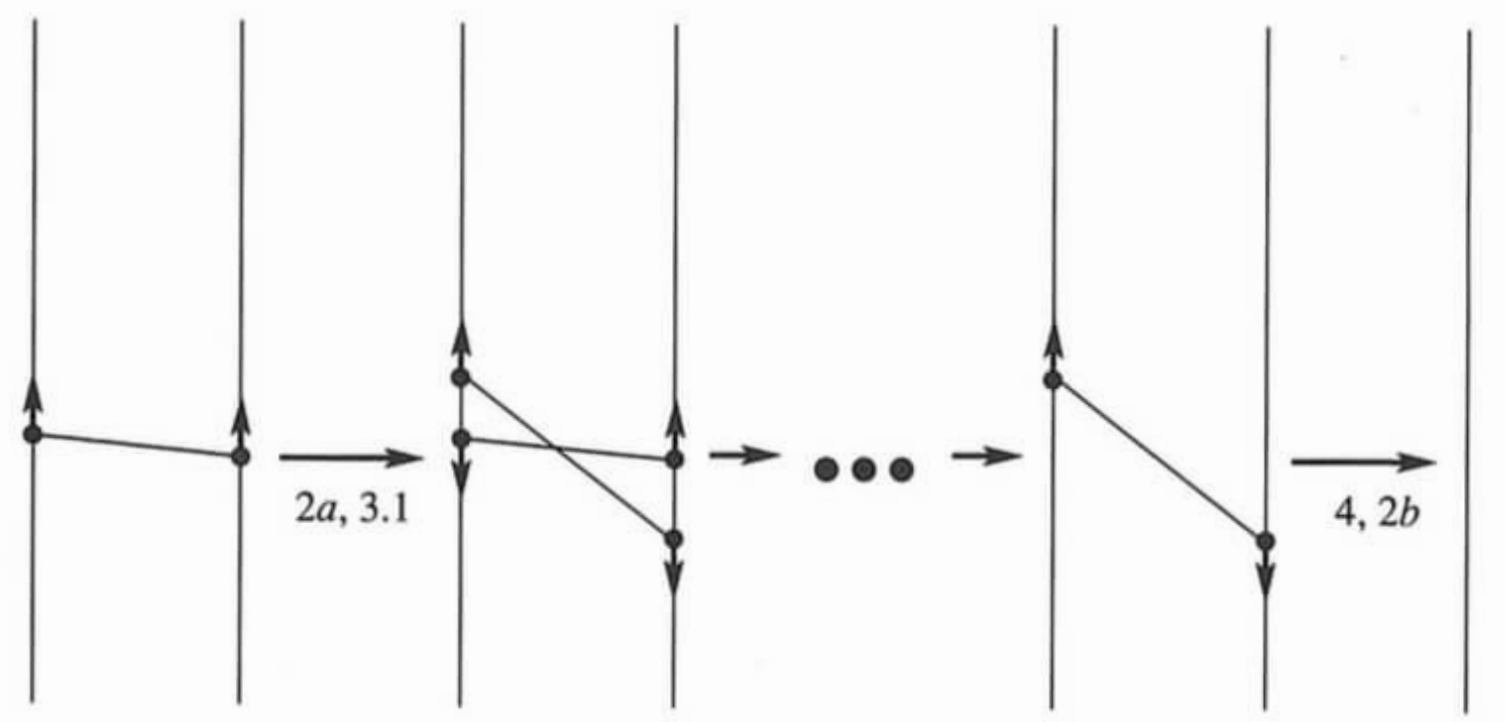

FIGURE 19

Consider a death of a pair of type 2. By Lemma 5 it dies together with a pair of type 1. The pair of type 1 either comes from the initial canonical form or appears together with a pair of type 2 as the result of a transformation of two pairs each consisting of two heights having the same directions (see Lemma 5). By Lemma 5, in the last case there is only one possible subsequence of metamorphoses, which is shown in Figure 20. In Figures 20-21, the numbers under the pointer denote the number of metamorphoses from Lemma 5 . The pointer without number denotes metamorphoses $2 \mathrm{a}$ or $2 \mathrm{~b}$.

The last situation on Figure 20 can be obtained from the initial situation by a simple shift of heights. We replace this subsequence by this shift of heights.

Consider a death of a pair of type 1. By Lemma 5, it dies either together 


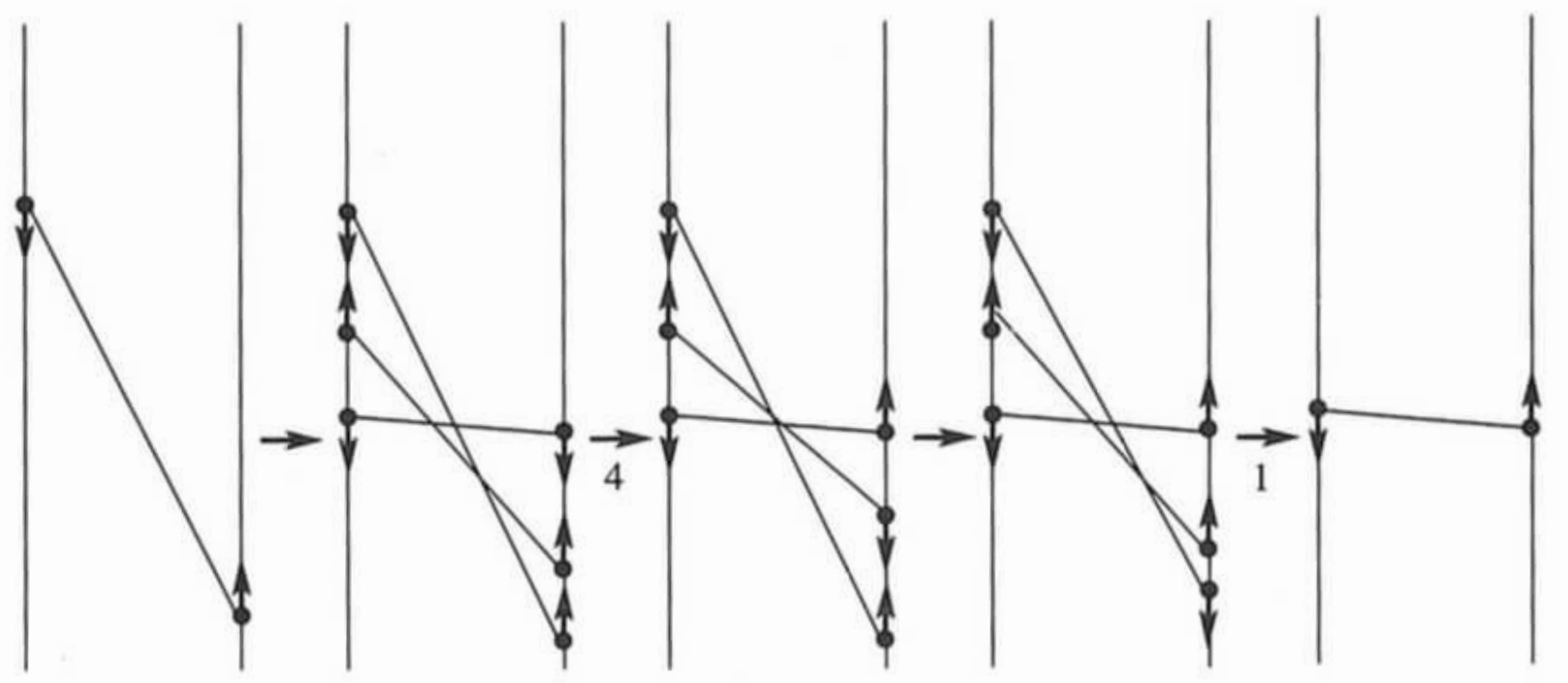

FIGURE 20

with a pair of type 2 or with a pair of type 1 . Consider the first case. If the pair of type 2 was not in the initial canonical form, then either it appears together with a pair of type 1 or type 2 or it is obtained from a pair of type 1 (see Lemma 5). The subsequence of metamorphoses is one of the only possible subsequences shown in Figure 21.

In the first case the last situation can be obtained from the initial situation by a shift of heights, in the second and the third case, by transforming a pair of type 1 to a pair of type 2 according to case 3 in Lemma 5 , in the fourth case the pairs of type 1 are directly reducible according to case 2 in Lemma 5.

The case when a pair of type 1 dies together with another pair of type 1 is similar to the previous ones. Consider the case in which a pair of type 1 is transformed into a pair of type 2 and then is reduced together with a pair of type 1. As shown in the previous section, in this case we can replace the subsequence of metamorphoses by reduction of pairs of type 1 according to case 2 in Lemma 5.

Thus we have replaced all subsequences of metamorphoses reducing pairs of types 1 or 2 by subsequences containing no metamorphoses $2 \mathrm{a}$. Delete all other metamorphoses $2 \mathrm{a}$ together with corresponding metamorphoses $2 \mathrm{~b}$ from the optimal sequence. We get an optimal sequence having no metamorphoses 2 a.

REMARK 5. If the given abstract FMC can be transformed by metamorphoses 1 and changes of signs of the generators of $C_{*}$ to a complex of simplest form (see §2.1), then any sequence of metamorphoses of the canonical form of the given abstract FMC is induced by a similar sequence of metamorphoses of the abstract FMC itself.

ExAMPLE. Let a function have the abstract FMC shown on Figure 22. Although it has ondex 0 , any smooth extension of the given function to the interior of the ball has one critical point of index 2 and one critical point of index 1 or index 3. 
1.

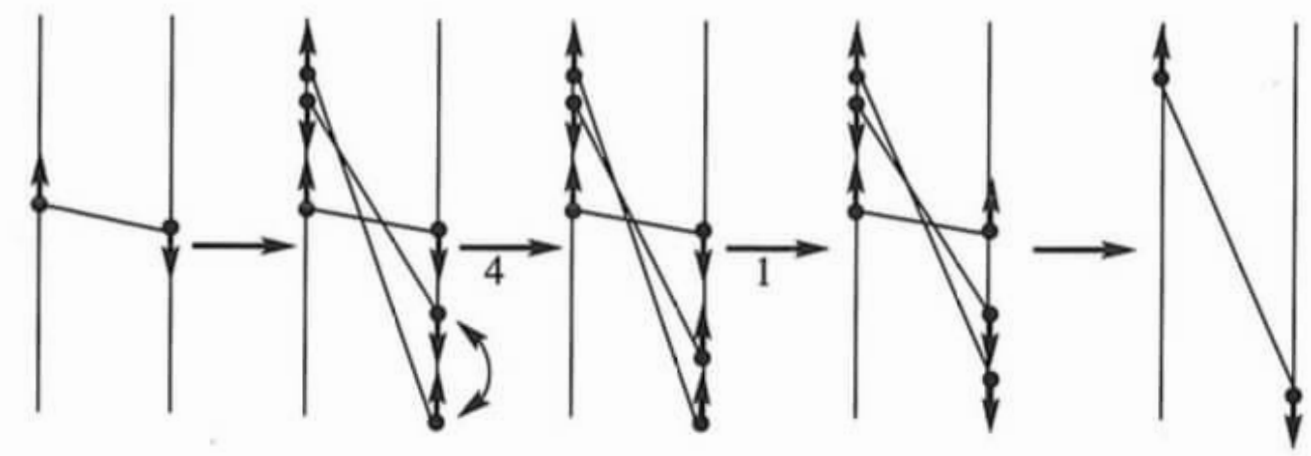

2.
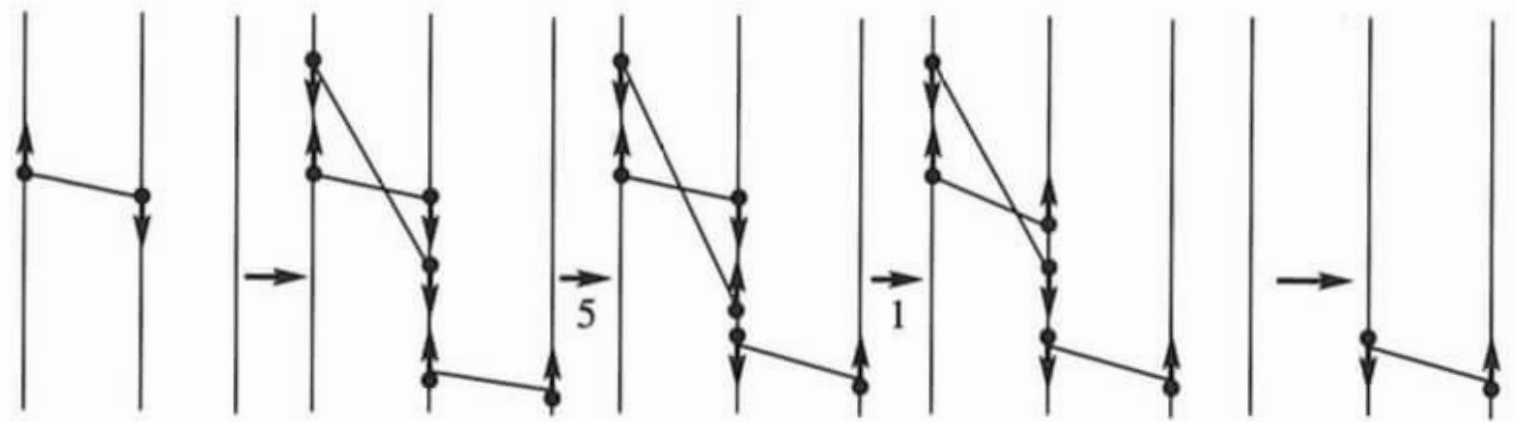

3.

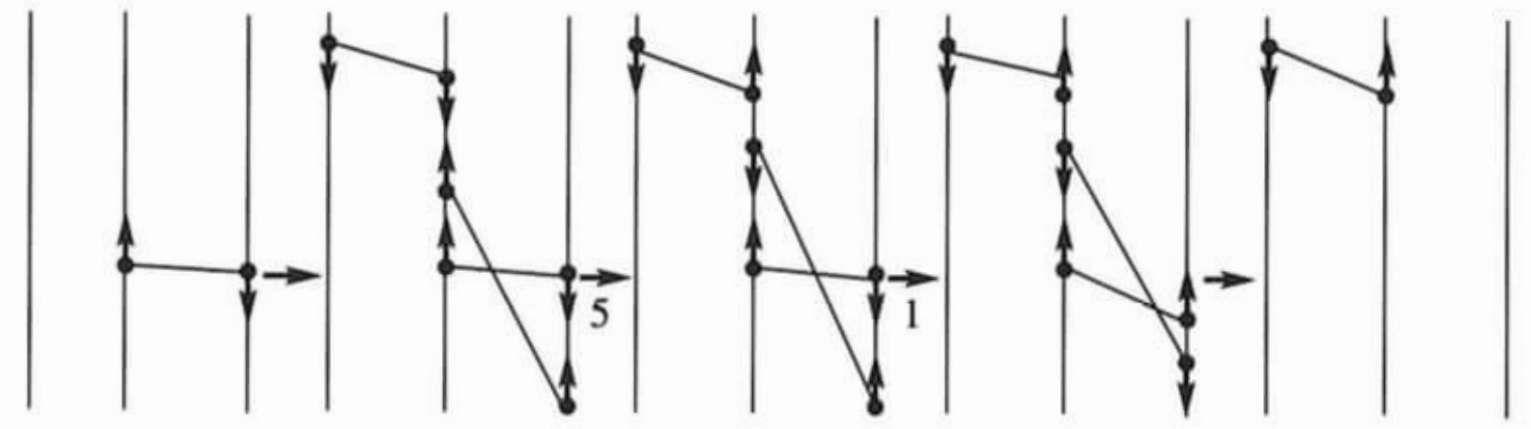

4.

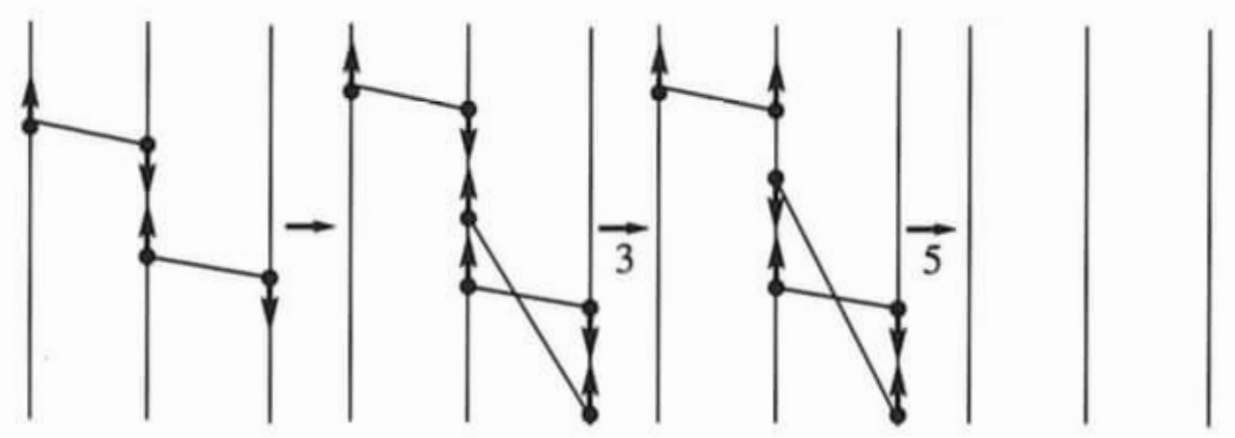

FIGURE 21

REMARK 6. The lower bounds given by the canonical forms over different fields can be different. Let a function has the abstract FMC shown on Figure 23. Then its canonical form over $\mathbb{Q}$ does not show any critical point, but the canonical form over $\mathbb{Z}_{2}$ shows that there are at least two critical points.

REMARK 7. Similar theorems hold for a smooth continuation of a given 

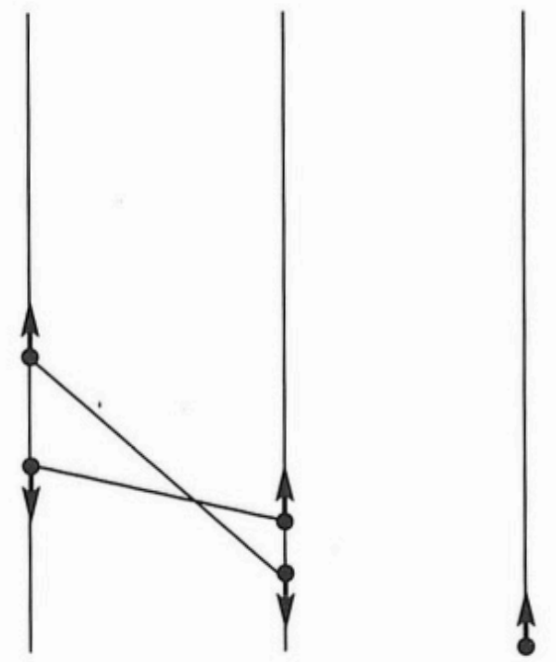

FIGURE 22

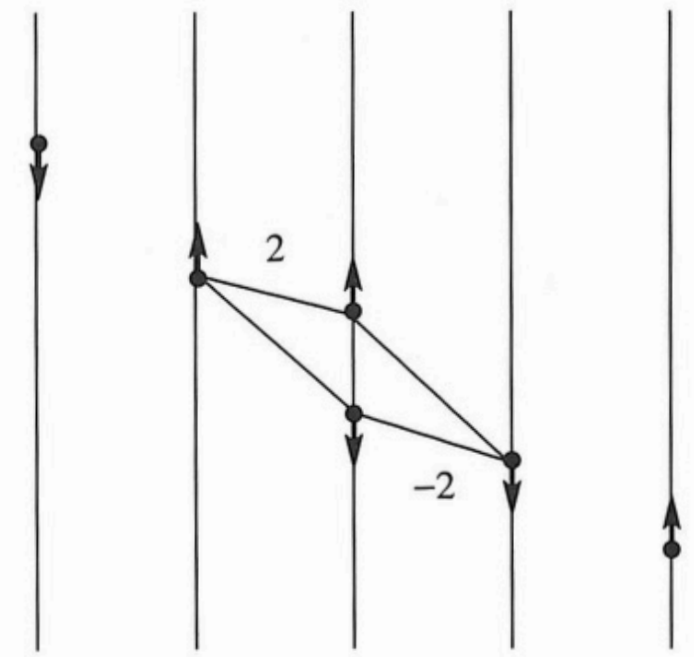

An abstract

FMC

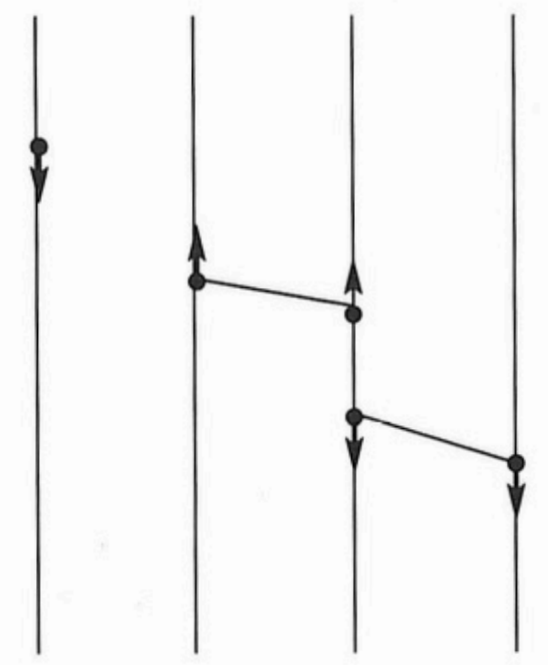

Its canonical form over $\mathbb{Q}$
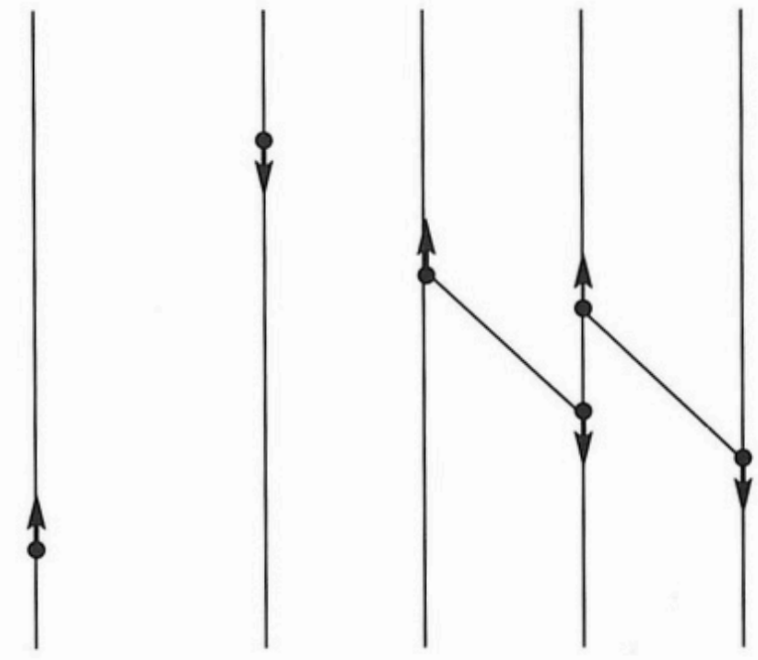

Its canonical form over $\mathbb{Z}_{2}$ 
function with possible degenerate critical points (see also Remark 2 after Theorem 2). They provide a lower bound for the number of critical points in this situation.

\section{§3. An application of similar invariants to functions on arbitrary manifold}

3.1. Invariants of connected components of the space of strictly Morse functions. Consider a generic smooth function on an arbitrary closed manifold. Consider the Morse complex for this function and a generic metric together with the critical values of the function. The Morse complex equipped with critical values of the function is the chain complex with ordered generators (see §2.1). Consider its canonical form over some field $F$. Recall that a function is said to be strictly Morse function if all its critical points are nondegenerate and all critical values are distinct.

THEOREM 4. The canonical form over any field corresponding to a strictly Morse function does not depend on the metric of the manifold. If $f_{1}, f_{2}$ are in the same connected component of the space of strictly Morse functions, then their canonical forms over an arbitrary field coincide.

Proof. The proof is similar to that of Lemma 3.

\section{REFERENCES}

1. J. Cerf, Stratification naturalle des espaces de fonctions differentiables réeles et le théorème de la pseudo-isotopie, Inst. Hautes Études Sci. Publ. Math. 39 (1970).

2. W. Klingenberg, The Morse complex, Simposia di Alta Matematika XXVI, Roma, 1982, pp. 117-122.

3. T. Matumoto, On the minimal ordered Morse functions on compact simply-connected manifolds, Publ. Res. Inst. Math. Sci. Kyoto Univ. 14 (1978), no. 3, 673-684.

4. J. Milnor, Lectures on the H-cobordism theorem, Princeton Univ. Press, Princeton, NJ, 1965.

5. M. Morse and G. B. Van Schaack, The critical point theory under general boundary conditions, Ann. Math. (2) 35 (1934), no. 3, 545-571.

6. V. V. Sharko, Functions on manifolds, "Naukova Dumka", Kiev, 1990; English transl., Amer. Math. Soc., Providence, RI, 1993.

7. R. Thom, Sur une partition en cellules associée à une fonction sur une variété, C. R. Acad. Sci. Paris 228 (1949), 973-975.

8. E. Witten, Supersymmetry and Morse theory, J. Differential Geometry 17 (1982), 661692.

Moscow State University, Moscow, Russia 